\title{
Existence of Weak Solutions for the Unsteady Interaction of a Viscous Fluid with an Elastic Plate
}

\author{
Céline Grandmont*
}

August 3, 2007

\begin{abstract}
We consider a three-dimensional viscous incompressible fluid governed by the Navier-Stokes equations, interacting with an elastic plate located on one part of the fluid boundary. We do not neglect the deformation of the fluid domain which consequently depends on the displacement of the structure. The purpose of this work is to study the solutions of this unsteady fluid-structure interaction problem, as the coefficient modeling the viscoelasticity (resp. the rotatory inertia) of the plate tends to zero. As a consequence, we obtain the existence of at least one weak solution for the limit problem (Navier-Stokes equation coupled with a plate in flexion) as long as the structure does not touch the bottom of the fluid cavity.
\end{abstract}

\section{Introduction.}

Many physical phenomena deal with a fluid interacting with a moving or deformable structure. This kind of problems have a lot of important applications, for instance in areolasticity, biomechanics, hydroelasticity, sedimentation... From the mathematical point of view they have been studied extensivelly over the last few years. Here we consider a viscous incompressible threedimensional fluid described by the Navier-Stokes equations interacting with a two-dimensional elastic plate in flexion. One already knows that if a viscous term or the rotatory inertia are taken into account in the plate equations, there exists at least one weak solution to this problem (see [4]). From the mechanical point of view adding a viscous term is a way to introduce dissipation in the plate model and from the mathematical point of view, this is a way to regularize the structure velocity. Here the dissipation coming from the fluid enables us to control the space high frequencies of the structure velocity and to pass to

*REO project, INRIA Rocquencourt, BP 10578153 Le Chesnay Cedex, France 
the limit in the coupled system as the additional viscous plate coefficient tends to zero, and thus obtain the existence of weak solutions of the limit problem. This limit system can also be obtained as the limit of the plate - Navier-Stokes system (with a regularized initial plate velocity) as the coefficient of the rotatory inertia tends to zero. In most of the previous studies the structure velocity is quite regular because of the model or because of the presence of a regularization operator in the equations. The existing results are concerned mainly with rigid body motions [5], [10], [11], [13], [16], [17], [18], [21], [22], [25], [24] or with the motion of a structure described by a finite number of modal functions [12] or a structure with additional "viscous" terms [2], [4], [8]. Recently, a significant breakthrough has been made by D. Coutand and S. Shkoller. In [6], [7] they prove the existence, locally in time, of a unique regular solution (assuming that the data are smooth enough and satisfy suitable compatibility conditions) for the Navier-Stokes equations coupled with linearized elasticity or quasi-linear elasticity. These are the only existence results where the full 3D elasticity is considered and that don't require additional viscous terms. Nevertheless, despite these new important results, the case of fluid-plate or fluid-shell interaction problems is not, as far as we know, solve. Here, taking advantage of the transverse motion of the plate, of the fact that the plate equations enable to have some regularity of the boundary of the fluid domain, we prove existence of weak solutions of a fluid-plate interaction problem. Even if we consider here a rather simple structure model, this is, to our knowledge, the first existence result of weak solutions in this direction. Note, moreover, that no compatibility assumptions are required and the existence result holds as long as the plate does not touch the bottom of the fluid cavity. Finally, the same results holds for a $2 \mathrm{D}$ viscous flow interacting with a one dimensional membrane.

In the first section we give the equations of the fluid-structure problem for which we derive a priori energy estimates. Next, definitions and properties of the energy spaces are detailed and the weak formulation of the problem is given. In particular, we build suitable extensions of the fluid test functions and liftings of the structure test functions. In the second section, we state our main results, the third section being devoted to the derivation of compactness properties that enable us to pass to the limit in the equations as the "viscous" plate coefficient tends to zero (Section 4).

\subsection{Presentation of the problem}

We assume that the fluid fills a three-dimensional cavity and interacts with a thin elastic structure, located on a part of the boundary of the fluid, the other part being rigid. For the sake of simplicity, we assume that, in the reference state, the elastic part of the fluid boundary is $\omega \times\{1\}$, where $\omega$ denotes a 
Lipschitz domain in $\mathbb{R}^{2}$. In the initial state the fluid occupies the domain $\Omega_{\eta_{I}}$ :

$$
\Omega_{\eta_{I}}=\left\{(x, y, z) \in \mathbb{R}^{3},(x, y) \in \omega, 0<z<1+\eta_{I}(x, y)\right\},
$$

where $\eta_{I}$ is a given initial displacement of the elastic part. The rigid part of $\partial \Omega_{\eta_{I}}$ is denoted by $\Gamma_{0}$. Note that we could also have considered the case of a fluid between two elastic plates or the case where $\omega \times\{1\}$ is a part of a smooth fluid domain boundary and obtained the same kind of results. We model the deformable part of the boundary by the classical linear plate theory for tranverse motions. We ignore in-plane motions. We take its edge to be clamped. We denote by $\eta_{\varepsilon}(t, x, y)$ the vertical displacement with respect to the rest configuration. The subscript $\varepsilon$ underlines the dependence of the solution with respect to the parameter $\varepsilon \geq 0$ which measures the "viscosity" of the plate (or the rotatory inertia). Then the equations describing the evolution of the transversal displacement $\eta_{\varepsilon}\left(\eta_{\varepsilon}=\eta_{\varepsilon}(t, x, y) \in \mathbb{R}\right)$ are

$$
\left\{\begin{array}{l}
\partial_{t t} \eta_{\varepsilon}+\Delta^{2} \eta_{\varepsilon}+\varepsilon \Delta^{2} \partial_{t} \eta_{\varepsilon}=g+\left(T_{f}^{\varepsilon}\right)_{3} \text { in } \omega \\
\eta_{\varepsilon}=\frac{\partial \eta_{\varepsilon}}{\partial n}=0 \text { on } \partial \omega \\
\eta_{\varepsilon}(0)=\eta_{I}, \partial_{t} \eta_{\varepsilon}(0)=\dot{\eta}_{I}
\end{array}\right.
$$

where $g$ denotes the given body force on the plate and $T_{f}^{\varepsilon}$ the surface force exerted by the fluid on the structure. The definition of $T_{f}^{\varepsilon}$ will be made precise later on. Instead of the additional viscosity term, we could have added $-\varepsilon \Delta \partial_{t t} \eta_{\varepsilon}$, which models the inertia of rotation. The domain occupied by the fluid at time $t$ is denoted by $\Omega_{\eta_{\varepsilon}}(t)$ :

$$
\Omega_{\eta_{\varepsilon}}(t)=\left\{(x, y, z) \in \mathbb{R}^{3},(x, y) \in \omega, 0<z<1+\eta_{\varepsilon}(t, x, y)\right\}
$$

The classical form of the governing equations for the fluid are

$$
\begin{cases}\partial_{t} \mathbf{u}_{\varepsilon}+\left(\mathbf{u}_{\varepsilon} \cdot \nabla\right) \mathbf{u}_{\varepsilon}-\nu \Delta \mathbf{u}_{\varepsilon}+\nabla p_{\varepsilon}=\mathbf{f} & \text { in } \Omega_{\eta_{\varepsilon}}(t), \\ \operatorname{div} \mathbf{u}_{\varepsilon}=0 & \text { in } \Omega_{\eta_{\varepsilon}}(t), \\ \mathbf{u}_{\varepsilon}(t, \cdot)=\mathbf{0} & \text { on } \Gamma_{0}, \\ \mathbf{u}_{\varepsilon}(0, \cdot)=\mathbf{u}_{I} & \text { in } \Omega_{\eta_{I}},\end{cases}
$$

where $\mathbf{u}_{\varepsilon}$ denotes the fluid velocity, and $p_{\varepsilon}$ the pressure field. The body force $\mathbf{f}$ and the initial velocity $\mathbf{u}_{I}$ are given.

Since the fluid is viscous, it adheres to the plate and thus the velocities coincide (in a sense to be defined) at the interface:

$$
\mathbf{u}_{\varepsilon}\left(t, x, y, 1+\eta_{\varepsilon}(t, x, y)\right)=\left(0,0, \partial_{t} \eta_{\varepsilon}(t, x, y)\right)^{T}, \quad(x, y) \in \omega .
$$

This condition, together with the incompressibility of the fluid leads to

$$
\int_{\omega} \partial_{t} \eta_{\varepsilon}=0
$$


Condition (5) states that the global volume of the cavity is preserved. The surface force $T_{f}$ exerted by the fluid on the plate can be defined by

$$
\int_{\omega} T_{f}^{\varepsilon} \cdot \overline{\mathbf{v}}=\int_{\partial \Omega_{\eta_{\varepsilon}}(t) \backslash \Gamma_{0}}\left(-2 \nu D\left(\mathbf{u}_{\varepsilon}\right) \cdot \mathbf{n}_{t}^{\varepsilon}+p_{\varepsilon} \mathbf{n}_{t}^{\varepsilon}\right) \cdot \mathbf{v} \quad \forall \mathbf{v},
$$

where $D\left(\mathbf{u}_{\varepsilon}\right)=\left(\nabla \mathbf{u}_{\varepsilon}+\left(\nabla \mathbf{u}_{\varepsilon}\right)^{T}\right) / 2$ is the strain tensor, $\mathbf{n}_{t}^{\varepsilon}$ denotes the outer unit normal at $\partial \Omega_{\eta_{\varepsilon}}(t) \backslash \Gamma_{0}\left(\mathbf{n}_{t}^{\varepsilon}=\frac{1}{\sqrt{1+\left(\partial_{x} \eta_{\varepsilon}\right)^{2}+\left(\partial_{y} \eta_{\varepsilon}\right)^{2}}}\left(-\partial_{x} \eta_{\varepsilon},-\partial_{y} \eta_{\varepsilon}, 1\right)^{T}\right)$ and $\overline{\mathbf{v}}(t, x, y)=\mathbf{v}\left(t, x, y, 1+\eta_{\varepsilon}(t, x, y)\right), \forall(x, y) \in \omega$. Note here that the pressure $p_{\varepsilon}$ is not defined up to a constant but is uniquely defined. Its average value ensures the global volume conservation of the fluid cavity. This average is in fact the Lagrange multiplier associated with the compatibility condition (5). Note that if Neumann boundary conditions had been imposed on $\Gamma_{0}$ then the plate displacement should not verify an additional "volume preserving" constraint. As noted in [4], the third component of $T_{f}^{\varepsilon}$ can be rewritten thanks to

$$
2\left(D\left(\mathbf{u}_{\varepsilon}\right) \cdot \mathbf{n}_{t}^{\varepsilon}\right)_{3}=\left(\nabla \mathbf{u}_{\varepsilon} \cdot \mathbf{n}_{t}^{\varepsilon}\right)_{3} .
$$

This simplification comes from the fact that the displacement at the fluidstructure interface is only transverse and from the incompressibility of the fluid. Thus $\forall \mathbf{v}$, such that $v_{i}\left(t, x, 1+\eta_{\varepsilon}(t, x, y)\right)=0, i=1,2,(t, x, y) \in(0, T) \times \omega$, we have

$$
\int_{\omega} T_{f}^{\varepsilon} \cdot \hat{\mathbf{v}}=\int_{\Gamma_{\eta_{\varepsilon}}(t)}\left(-\nu \nabla \mathbf{u}_{\varepsilon} \cdot \mathbf{n}_{t}^{\varepsilon}+p_{\varepsilon} \mathbf{n}_{t}^{\varepsilon}\right)_{3} v_{3}
$$

\section{$1.2 \quad$ A priori Estimates}

In this subsection we recall the a priori estimates satisfied by any solution, assuming that it is smooth enough. We multiply the Navier-Stokes equations by $\mathbf{u}_{\varepsilon}$ and integrate over $\Omega_{\eta_{\varepsilon}}(t)$, and we multiply the plate equations by $\partial_{t} \eta_{\varepsilon}$ and integrate over $\omega$ and add these two contributions. After integrations by parts and taking into account the coupling conditions (equality of the velocities (4), and the definition of $\left.T_{f}^{\varepsilon}(7)\right)$ we obtain the following energy equality:

$$
\begin{aligned}
& \frac{1}{2} \frac{d}{d t} \int_{\Omega_{\eta_{\varepsilon}}(t)}\left|\mathbf{u}_{\varepsilon}\right|^{2}+2 \nu \int_{\Omega_{\eta_{\varepsilon}}(t)}\left|\nabla\left(\mathbf{u}_{\varepsilon}\right)\right|^{2} \\
& +\frac{1}{2} \frac{d}{d t} \int_{\omega}\left(\partial_{t} \eta_{\varepsilon}\right)^{2}+\frac{1}{2} \frac{d}{d t} \int_{\omega}\left(\Delta \eta_{\varepsilon}\right)^{2}+\varepsilon \int_{\omega}\left(\Delta \partial_{t} \eta_{\varepsilon}\right)^{2} \\
& =\int_{\Omega_{\eta_{\varepsilon}}(t)} \mathbf{f} \cdot \mathbf{u}_{\varepsilon}+\int_{\omega} g \partial_{t} \eta_{\varepsilon} .
\end{aligned}
$$

Hence, using Cauchy-Schwarz and Young's inequalities and the Gronwall's lemma: 


$$
\begin{aligned}
& \frac{1}{2}\left\|\mathbf{u}_{\varepsilon}(t, \cdot)\right\|_{L^{2}\left(\Omega_{\eta_{\varepsilon}}(t)\right)}^{2}+2 \nu \int_{0}^{t}\left\|\nabla\left(\mathbf{u}_{\varepsilon}\right)(s, \cdot)\right\|_{L^{2}\left(\Omega_{\eta_{\varepsilon}}(s)\right)}^{2} d s \\
& +\frac{1}{2}\left\|\partial_{t} \eta_{\varepsilon}(t, \cdot)\right\|_{L^{2}(\omega)}^{2}+\frac{1}{2}\left\|\Delta \eta_{\varepsilon}(t, \cdot)\right\|_{L^{2}(\omega)}^{2}+\varepsilon \int_{0}^{t}\left\|\Delta \partial_{t} \eta_{\varepsilon}(s, \cdot)\right\|_{L^{2}(\omega)}^{2} d s \\
& \leq e^{t}\left(\frac{1}{2}\left\|\mathbf{u}_{I}\right\|_{L^{2}\left(\Omega_{\eta_{I}}\right)}^{2}+\frac{1}{2}\left\|\dot{\eta}_{I}\right\|_{L^{2}(\omega)}^{2}+\frac{1}{2}\left\|\Delta \eta_{I}\right\|_{L^{2}(\omega)}^{2}\right) \\
& +\frac{1}{2} \int_{0}^{t} \exp (t-s)\left(\|\mathbf{f}(s, \cdot)\|_{L^{2}\left(\Omega_{\eta_{\varepsilon}}(s)\right)}^{2}+\|g(s, \cdot)\|_{L^{2}(\omega)}^{2}\right) d s .
\end{aligned}
$$

Thus, assuming that $\mathbf{f} \in L^{2}\left(0, T ; L^{2}\left(\mathbb{R}^{3}\right)\right), g \in L^{2}\left(0, T ; L^{2}(\omega)\right), \mathbf{u}_{I} \in L^{2}\left(\Omega_{\eta_{I}}\right)$, $\eta_{I} \in H_{0}^{2}(\omega), \dot{\eta}_{I} \in L^{2}(\omega)$,

$\mathbf{u}_{\varepsilon}$ is bounded, uniformly in $\varepsilon$, in $L^{\infty}\left(0, T ; L^{2}\left(\Omega_{\eta_{\varepsilon}}(t)\right)\right)$,

$\nabla \mathbf{u}_{\varepsilon}$ is bounded, uniformly in $\varepsilon$, in $L^{2}\left(0, T ; L^{2}\left(\Omega_{\eta_{\varepsilon}}(t)\right)\right)$,

and

$\eta_{\varepsilon}$ is bounded, uniformly in $\varepsilon$, in $W^{1, \infty}\left(0, T ; L^{2}(\omega)\right) \cap L^{\infty}\left(0, T ; H_{0}^{2} \cdot(\omega)\right)$,

Moreover, if $\varepsilon>0$,

$$
\partial_{t} \eta_{\varepsilon} \in L^{2}\left(0, T ; H_{0}^{2}(\omega)\right) .
$$

Consequently the spaces $L^{p}\left(0, T ; L^{2}\left(\Omega_{\gamma}(t)\right)\right), L^{2}\left(0, T ; H^{1}\left(\Omega_{\gamma}(t)\right)\right)$ need to be defined, for $\gamma$ belonging to $W^{1, \infty}\left(0, T ; L^{2}(\omega)\right) \cap L^{\infty}\left(0, T ; H_{0}^{2}(\omega)\right)$. Note that the following continuous injection holds:

$$
W^{1, \infty}\left(0, T ; L^{2}(\omega)\right) \cap L^{\infty}\left(0, T ; H^{2}(\omega)\right) \hookrightarrow C^{0,1-\theta}\left([0, T] ; H^{2 \theta}(\omega)\right),
$$

for all $0<\theta<1$. In particular,

$$
W^{1, \infty}\left(0, T ; L^{2}(\omega)\right) \cap L^{\infty}\left(0, T ; H^{2}(\omega)\right) \hookrightarrow C^{0, \mu}\left([0, T] ; C^{0,1 / 2-\mu}(\bar{\omega})\right),
$$

for all $0<\mu<1 / 2$. The proof of the first injection relies on standard hilbertian interpolation inequalities (see [20]). The other is deduced from the first one and from Sobolev injections in dimension two (see [3]). Consequently, this displacement regularity does not imply that the fluid domain boundary is Lipschitz and we have to pay a special attention to the definitions of the functional spaces. We have also to give a sense to the equality of the velocities. Thus we are going to give some technical lemmas, definitions and properties, most of which can be found in [4].

\subsection{Preliminary definitions and properties}

We now turn to the definition of some functional spaces. These definitions can be found in [4], but for the sake of completness we recall them here. Let 
$T>0$ and $\delta$ belong to $C^{0}([0, T] \times \bar{\omega})$ such that for some positive $M$ and $\alpha$, $M \geq 1+\delta(t, x, y) \geq \alpha>0$ for all $(t, x, y) \in[0, T] \times \bar{\omega}$, and such that $\delta=0$ on $\partial \omega$. The set $\Omega_{\delta}(t)$ defined by

$$
\Omega_{\delta}(t)=\left\{(x, y, z) \in \mathbb{R}^{3},(x, y) \in \omega, 0<z<1+\delta(t, x, y)\right\},
$$

is an open subset of $\mathbb{R}^{3}$ for every $t \in[0, T]$ which is included in $\mathcal{C}_{M}=\omega \times(0, M)$. Let $\widehat{\Omega}_{\delta}$ be the open domain of $\mathbb{R}^{4}$ defined by

$$
\widehat{\Omega}_{\delta}=\bigcup_{t \in(0, T)}\{t\} \times \Omega_{\delta}(t) .
$$

We set $\widehat{\mathcal{C}_{M}}=(0, T) \times \mathcal{C}_{M}$. One can define in a standard way the spaces $L^{p}\left(\Omega_{\delta}(t)\right)$, $H^{1}\left(\Omega_{\delta}(t)\right), H_{0}^{1}\left(\Omega_{\delta}(t)\right)$, for every $t$, and $L^{p}\left(\widehat{\Omega}_{\delta}\right), H^{1}\left(\widehat{\Omega}_{\delta}\right), L^{p}\left(\widehat{\mathcal{C}_{M}}\right), H^{1}\left(\widehat{\mathcal{C}_{M}}\right) \ldots$ The space $H_{0, \Gamma_{0}}^{1}\left(\Omega_{\delta}(t)\right)$ will denote the subspace of $H_{0, \Gamma_{0}}^{1}\left(\Omega_{\delta}(t)\right)$ of functions of zero trace on $\Gamma_{0}=\omega \times\{0\} \cup \partial \omega \times(0,1)$. We then define:

$$
\begin{gathered}
L^{2}\left(0, T ; H^{1}\left(\Omega_{\delta}(t)\right)\right)=\left\{v \in L^{2}\left(\widehat{\Omega}_{\delta}\right), \nabla v \in L^{2}\left(\widehat{\Omega}_{\delta}\right)\right\}, \\
L^{2}\left(0, T ; H_{0}^{1}\left(\Omega_{\delta}(t)\right)\right)=\overline{\mathcal{D}\left(\widehat{\Omega}_{\delta}\right)}{ }^{2}\left(0, T ; H^{1}\left(\Omega_{\delta}(t)\right)\right) \\
\mathcal{V}_{\delta}=\left\{\mathbf{v} \in C^{1}\left(\overline{\widehat{\Omega}_{\delta}}\right), \operatorname{div} \mathbf{v}=0, \mathbf{v}=\mathbf{0} \text { on }(0, T) \times \Gamma_{0}\right\}, \\
V_{\delta}={\overline{\mathcal{V}_{\delta}}}^{L^{2}\left(0, T ; H^{1}\left(\Omega_{\delta}(t)\right)\right)},
\end{gathered}
$$

and

$$
L^{\infty}\left(0, T ; L^{2}\left(\Omega_{\delta}(t)\right)\right)=\left\{v \in L^{2}\left(\widehat{\Omega}_{\delta}\right), \sup \operatorname{ess}_{t \in(0, T)}\|v\|_{L^{2}\left(\Omega_{\delta}(t)\right)}<+\infty\right\} .
$$

Moreover we denote

$$
V=\left\{\mathbf{v} \in L^{2}\left(0, T ; H^{1}\left(\mathcal{C}_{M}\right)\right), \operatorname{div} \mathbf{v}=0, \mathbf{v}=\mathbf{0} \text { on }(0, T) \times\left(\Gamma_{0} \cup \Gamma_{1}\right)\right\},
$$

where $\Gamma_{1}=\partial \omega \times(1, M)$.

The space $V_{\delta}$ can be characterized as follows:

$$
V_{\delta}=\left\{\mathbf{v} \in L^{2}\left(0, T ; H^{1}\left(\Omega_{\delta}(t)\right)\right), \operatorname{div} \mathbf{v}=0, \mathbf{v}=\mathbf{0} \text { on }(0, T) \times \Gamma_{0}\right\} .
$$

In the case of a Lipschitz or a star-shaped domain independent of time this follows from standard arguments (see [26] or [15]). In our case it can be proved using the fact that the domain $\Omega_{\delta}(t)$ is locally a subgraph. This property will be extensively used in all what follows.

Next we recall various lemma that explain how the trace on $\partial \Omega_{\delta}(t) \backslash \Gamma_{0}$ makes sense and define extension and lifting operators, explore some properties of the spaces defined above... We omit the proves whenever they can be found 
in [4]. Note that these results take advantage of the fact that the fluid domain is a subgraph because the displacement of the interface is only transverse.

Let us consider the linear mapping $\gamma_{\delta(t)}: v \mapsto v(x, y, 1+\delta(t, x, y))$ defined for $v \in C^{0}\left(\bar{\Omega}_{\delta}(t)\right)$.

Lemma 1 For every $t \in[0, T]$, the mapping $\gamma_{\delta(t)}$ from $C^{1}\left(\overline{\mathcal{C}_{M}}\right)\left(\right.$ resp. $\left.C^{1}\left(\bar{\Omega}_{\delta}(t)\right)\right)$ in $C^{0}(\bar{\omega})$ can be extended by continuity to a linear mapping from $H^{1}\left(\mathcal{C}_{M}\right)$ (resp. $\left.H^{1}\left(\Omega_{\delta}(t)\right)\right)$ into $L^{2}(\omega)$.

Corollary 1 If $v \in L^{2}\left(0, T ; H^{1}\left(\Omega_{\delta}(t)\right)\right)$ then $\gamma_{\delta(t)}(v) \in L^{2}\left(0, T ; L^{2}(\omega)\right)$.

Thus, the trace of $v(x, y, 1+\delta(t, x, y))$ on $\omega$ makes sense at least in $L^{2}(\omega)$. The following lemma precises the regurarity of $\gamma_{\delta(t)}(v)$ when assuming moreover that $\delta$ belongs to $L^{\infty}\left(0, T ; H_{0}^{2}(\omega)\right)$. This additional regularity will play a crucial role in our asymptotic study and will enable us to control the space high frequencies of the structure velocity.

Lemma 2 Assuming that $\delta \in C^{0}\left([0, T] ; C^{0}(\bar{\omega})\right) \cap L^{\infty}\left(0, T ; H_{0}^{2}(\omega)\right)$ then, for any $v \in H^{1}\left(\Omega_{\delta}(t)\right), \gamma_{\delta(t)}(v) \in W^{1-1 / p, p}(\omega), \forall 1<p<2$ and for $\frac{3}{2} \leq p<2$, $\gamma_{\delta(t)}(v) \in H^{\frac{3 p-2}{p}}(\omega)$, for a.e. $t$. If $v \in L^{2}\left(0, T ; H^{1}\left(\Omega_{\delta}(t)\right)\right)$ then $\gamma_{\delta(t)}(v) \in$ $L^{2}\left(0, T ; W^{1-1 / p, p}(\omega)\right), \forall 1<p<2$ and $\gamma_{\delta(t)}(v) \in L^{2}\left(0, T ; H^{\frac{3 p-2}{p}}(\omega)\right), \forall \frac{3}{2} \leq p<$ 2 .

Proof: Let us define an auxilary function by

$$
w(t, x, y, s)=v(x, y, 1+\delta(t, x, y)-s), 0 \leq s \leq \alpha .
$$

It is clear that $w$ belongs to $L^{2}\left(\mathcal{C}_{\alpha}\right), \forall t$. Moreover

$\nabla w(t, x, y, s)=\left(\begin{array}{c}\partial_{x} v(x, y, 1+\delta(t, x, y)-s)+\partial_{x} \delta(t, x, y) \partial_{z} v(x, y, 1+\delta(t, x, y)-s) \\ \partial_{y} v(x, y, 1+\delta(t, x, y)-s)+\partial_{y} \delta(x, y) \partial_{z} v(x, y, 1+\delta(t, x, y)-s), \\ -\partial_{z} v(x, y, 1+\delta(t, x, y)-s)\end{array}\right)$.

Since $H^{1}(\omega)$ is continuously imbedded in $L^{q}(\omega), \forall q<\infty$ and $\partial_{i} v(x, y, 1+$ $\delta(t, x, y)-s) \in L^{2}\left(\mathcal{C}_{\alpha}\right) \forall t$, we deduce that $\nabla w \in L^{p}\left(\mathcal{C}_{\alpha}\right)$ for all $1<p<2$, for a. e. $t$. Thus $w \in W^{1, p}\left(\mathcal{C}_{\alpha}\right)$, and $\gamma_{\delta(t)}(v)=\left.w\right|_{s=0} \in W^{1-1 / p, p}(\omega), \forall 1<p<2$ for a. e. $t$. Moreover, for a. e. $t$,

$$
\left\|\gamma_{\delta(t)}(v)\right\|_{W^{1-1 / p, p}(\omega)} \leq C\left(\|\delta\|_{C^{0}\left([0, T] ; C^{0}(\bar{\omega})\right) \cap L^{\infty}\left(0, T ; H_{0}^{2}(\omega)\right)},\|v\|_{H^{1}\left(\Omega_{\delta}(t)\right)}\right) .
$$

Furthermore, from Sobolev injections (see [1], Th. 7.58, p. 218), we deduce that for $2>p \geq \frac{3}{2}, \gamma_{\delta(t)}(v) \in H^{\frac{3 p-2}{p}}(\omega)$ and

$$
\left\|\gamma_{\delta(t)}(v)\right\|_{H^{\frac{3 p-2}{p}}(\omega)} \leq C\left(\|\delta\|_{C^{0}\left([0, T] ; C^{0}(\bar{\omega})\right) \cap L^{\infty}\left(0, T ; H_{0}^{2}(\omega)\right)},\|v\|_{H^{1}\left(\Omega_{\delta}(t)\right)}\right) .
$$

Now we are going to give a characterization of $H_{0}^{1}\left(\Omega_{\delta}(t)\right)$ with the help of the mapping $\gamma_{\delta(t)}$. An additional assumption on the boundary displacement $\delta$ is needed: $\delta$ is assumed to belong to $C^{0}\left([0, T] ; H^{1}(\omega)\right)$ (this is not an optimal assumption). 
Lemma 3 Assuming that $\delta \in C^{0}\left([0, T] ; C^{0}(\bar{\omega}) \cap H^{1}(\omega)\right)$ then

$$
H_{0}^{1}\left(\Omega_{\delta}(t)\right)=\left\{v \in H_{0, \Gamma_{0}}^{1}\left(\Omega_{\delta}(t)\right), \gamma_{\delta(t)}(v)=0\right\}
$$

Corollary 2 If $v \in L^{2}\left(0, T ; H_{0, \Gamma_{0}}^{1}\left(\Omega_{\delta}(t)\right)\right)$ and $\gamma_{\delta(t)}(v)=0$, for a.e. $t$, then $v \in L^{2}\left(0, T ; H_{0}^{1}\left(\Omega_{\delta}(t)\right)\right)$, and the converse is also true.

We now state a lemma that enables to extend a function $\mathbf{v} \in V_{\delta}$ such that $\gamma_{\delta(t)}(\mathbf{v})=(0,0, b)^{T}, b \in L^{2}\left(0, T ; H^{1}(\omega)\right)$, the extension belonging to $V$.

Lemma 4 We assume that $\delta \in C^{0}\left([0, T] ; C^{0}(\bar{\omega}) \cap H_{0}^{1}(\omega)\right)$. Let $\mathbf{v} \in V_{\delta}$, such that, for a.e. $t, \gamma_{\delta(t)}(\mathbf{v})=(0,0, b)^{T}, b \in L^{2}\left(0, T ; H_{0}^{1}(\omega)\right)$. The function defined by

$$
\overline{\mathbf{v}}=\mid \begin{aligned}
& \mathbf{v} \text { in } \widehat{\Omega}_{\delta} \\
& (0,0, b)^{T} \text { in } \widehat{\mathcal{C}_{M}} \backslash \widehat{\Omega}_{\delta}
\end{aligned}
$$

belongs to $V$, and

$$
\|\overline{\mathbf{v}}\|_{V} \leq C\left(\|\mathbf{v}\|_{V_{\delta}}+\|b\|_{L^{2}\left(0, T ; H^{1}(\omega)\right)}\right)
$$

where $C$ depends only on $M$.

Remark 1 If $\mathbf{v} \in L^{\infty}\left(0, T ; L^{2}\left(\Omega_{\delta}(t)\right)\right)$ and $b \in L^{\infty}\left(0, T ; L^{2}(\omega)\right)$ then $\overline{\mathbf{v}} \in$ $L^{\infty}\left(0, T ; L^{2}\left(\mathcal{C}_{M}\right)\right)$.

Next we build different lifting operators:

Lemma 5 For every $\phi \in H_{0}^{1}(\omega)$ there exists $w \in H_{0, \Gamma_{0}}^{1}\left(\Omega_{\delta}(t)\right)$ such that

$$
\gamma_{\delta(t)}(w)=\phi \quad \text { and } \quad\|w\|_{H^{1}\left(\Omega_{\delta}(t)\right)} \leq C_{\alpha}\|\phi\|_{H^{1}(\omega)}
$$

For every $b \in H_{0}^{1}(\omega)$ such that $\int_{\omega} b=0$ there exists $\mathbf{v}$ such that $\gamma_{\delta(t)}(\mathbf{v})=$ $(0,0, b)^{T}, \operatorname{div}(\mathbf{v})=0$ and

$$
\|\mathbf{v}\|_{H_{0, \Gamma_{0}}^{1}\left(\Omega_{\delta}(t)\right)} \leq C_{\alpha}\|b\|_{H_{0}^{1}(\omega)} .
$$

Proof: Indeed

$$
w=\mid \begin{aligned}
& \phi \text { in } \Omega_{\delta}(t) \backslash \mathcal{C}_{\alpha} \\
& \mathcal{R}\left(\frac{z}{\alpha} \phi\right) \text { in } \mathcal{C}_{\alpha},
\end{aligned}
$$

where $\mathcal{R}$ is a continuous lifting operator from $H^{1 / 2}\left(\partial \mathcal{C}_{\alpha}\right)$ into $H^{1}\left(\mathcal{C}_{\alpha}\right)$ and $\mathcal{C}_{\alpha}=$ $\omega \times(0, \alpha)$, verifies the desired properties. Moreover, if we consider $b \in H_{0}^{1}(\omega)$ such that $\int_{\omega} b=0$ then, $\tilde{\mathcal{R}}$ is a continuous lifting operator from $H^{1 / 2}\left(\partial \mathcal{C}_{\alpha}\right)$ into $H^{1}\left(\mathcal{C}_{\alpha}\right)$ such that $\operatorname{div}(\tilde{\mathcal{R}} \mathbf{v})=0$

$$
\mathbf{v}=\mid \begin{aligned}
& (0,0, b)^{T} \text { in } \Omega_{\delta}(t) \backslash \mathcal{C}_{\alpha} \\
& \tilde{\mathcal{R}}\left(0,0, \frac{z}{\alpha} b\right)^{T} \text { in } \mathcal{C}_{\alpha},
\end{aligned}
$$


is divergence free and belongs to $H_{0, \Gamma_{0}}^{1}\left(\Omega_{\delta}(t)\right)$. Furthermore, we have for a. e. $t$

$$
\|\mathbf{v}\|_{H_{0, \Gamma_{0}}^{1}\left(\Omega_{\delta}(t)\right)} \leq C_{\alpha}\|b\|_{H_{0}^{1}(\omega)} .
$$

Consequently (16) defines a continuous linear lifting from $\left\{b \in H_{0}^{1}(\omega)\right.$, s.t. $\int_{\omega} b=$ $0\}$ into $\left\{\mathbf{v} \in H_{0, \Gamma_{0}}^{1}\left(\Omega_{\delta}(t)\right)\right.$, s. t. $\left.\operatorname{div}(\tilde{\mathcal{R}} \mathbf{v})=0\right\}$.

Remark 2 Thanks to the previous lemma the space

$$
\left\{\mathbf{v} \in V_{\delta}, \gamma_{\delta(t)}(\mathbf{v})=(0,0, b)^{T}, \text { for a.e. } t, b \in L^{2}\left(0, T ; H_{0}^{1}(\omega)\right)\right\},
$$

is equal to the sum of the two following spaces:

$$
\overline{\left\{\mathbf{v} \in \mathcal{D}\left(\widehat{\Omega}_{\delta}\right), \operatorname{div} \mathbf{v}=0\right\}}{ }^{L^{2}\left(0, T ; H^{1}\left(\Omega_{\delta}(t)\right)\right.},
$$

and

$$
\left\{\mathbf{v}, \mathbf{v}=\mid \begin{array}{l}
(0,0, b)^{T} \text { in } \Omega_{\delta}(t) \backslash \mathcal{C}_{\alpha} \\
\tilde{\mathcal{R}}\left(0,0, \frac{z}{\alpha} b\right)^{T} \text { in } \mathcal{C}_{\alpha},
\end{array} \text { for a.e. } t, b \in L^{2}\left(0, T ; H_{0}^{1}(\omega)\right), \int_{\omega} b=0 .\right\}
$$

We also need to build a "lifting" operator of $(0,0, b)^{T}$ for any $b$ that belongs only to $H^{s}(\omega), 0 \leq s<\frac{1}{2}$ since the structure velocity $\partial_{t} \eta_{\varepsilon}$ will be bounded, uniformly in $\varepsilon$, only in $L^{2}\left(0, T ; H^{s}(\omega)\right), \forall 0 \leq s<\frac{1}{2}$ and not in $L^{2}\left(0, T ; H_{0}^{1}(\omega)\right)$ (see Lemma 2).

Lemma 6 For all $b \in L^{2}\left(0, T ; H^{s}(\omega)\right), 0 \leq s<\frac{1}{2}$ such that $\int_{\omega} b=0$, there exists a lifting operator $\mathcal{R}_{\alpha}$ satisfing $\gamma_{\delta(t)}\left(\mathcal{R}_{\alpha}(b)\right)=(0,0, b)^{T}$ and $\operatorname{div}\left(\mathcal{R}_{\alpha}(b)\right)=$ 0 and for a. e. $t$

$$
\left\|\mathcal{R}_{\alpha}(b)\right\|_{H^{s}\left(\mathcal{C}_{M}\right)} \leq C\|b\|_{H^{s}(\omega)}, \forall 0 \leq s<\frac{1}{2} .
$$

Proof: A rather naive construction will satisfy the desired properties. Let us define $\mathcal{R}_{\alpha}$ by

$$
\mathcal{R}_{\alpha}(b)=\mid \begin{aligned}
& (0,0, b)^{T} \text { for } z \geq \alpha, \\
& \left(0,0, \frac{z}{\alpha} b\right)^{T}+\mathbf{w}_{\alpha} \text { in } \mathcal{C}_{\alpha}, \quad \text { for a. e. } t,
\end{aligned}
$$

where $\mathbf{w}_{\alpha}$ is such that $\operatorname{div}\left(\mathbf{w}_{\alpha}\right)=b$ and $\mathbf{w}_{\alpha} \in H_{0}^{1}\left(\mathcal{C}_{\alpha}\right),\left\|\mathbf{w}_{\alpha}\right\|_{H_{0}^{1}\left(\mathcal{C}_{\alpha}\right)} \leq C\|b\|_{L^{2}(\omega)}$, for a. e. $t$. Such a function exists (see for instance [14]). It is easy to see that $\mathcal{R}_{\alpha}(b)$ is divergence free. Moreover, $\mathcal{R}_{\alpha}$ is linear continuous from $L^{2}\left(0, T, L^{2}(\omega)\right)$ (resp. $\left.L^{2}\left(0, T, H_{0}^{1}(\omega)\right)\right)$ into $L^{2}\left(0, T, L^{2}\left(\mathcal{C}_{M}\right)\right)\left(\right.$ resp. $\left.L^{2}\left(0, T, H_{0, \Gamma_{0}}^{1}\left(\mathcal{C}_{M}\right)\right)\right)$, thus, by interpolation (see [19]), $\mathcal{R}_{\alpha}$ is linear continuous from $\left.L^{2}\left(0, T, H^{s}(\omega)\right)\right)$ into $L^{2}\left(0, T, H^{s}\left(\mathcal{C}_{M}\right)\right), \forall 0 \leq s<\frac{1}{2}$. Consequently, (17) holds true.

Remark 3 The trace $\gamma_{\delta(t)}\left(\mathcal{R}_{\alpha}(b)\right)$ makes sense for any $b \in L^{2}(\omega)$ since $\mathcal{R}_{\alpha}(b)$ is regular enough with respect to $z$. 
We end this section by mentionning that Korn's and Poincaré's inequalities hold in the considered spaces.

Lemma 7 For all $\mathbf{u}$ and $\mathbf{v}$ in

$$
\left\{\mathbf{v} \in V_{\delta}, \exists b \in L^{2}\left(0, T ; H_{0}^{1}(\omega)\right), \gamma_{\delta(t)}(\mathbf{v})=(0,0, b)^{T} \text { for a.e. } t\right\}
$$

we have

$$
2 \int_{\Omega_{\delta}(t)} D(\mathbf{u}): D(\mathbf{v})=\int_{\Omega_{\delta}(t)} \nabla \mathbf{u}: \nabla \mathbf{v}, \text { for a.e. } t,
$$

and consequently the following Korn's "equality" holds:

$$
\sqrt{2}\|D(\mathbf{u})\|_{L^{2}\left(\Omega_{\delta(t)}\right)}=\|\nabla \mathbf{u}\|_{L^{2}\left(\Omega_{\delta(t)}\right)}, \text { for a.e. } t .
$$

Lemma 8 Let $v \in H_{0, \Gamma_{0}}^{1}\left(\Omega_{\delta}(t)\right)$, then

$$
\|v\|_{L^{2}\left(\Omega_{\delta}(t)\right)} \leq M\|\nabla v\|_{L^{2}\left(\Omega_{\delta}(t)\right)} .
$$

\subsection{Weak formulation}

Let $\eta_{I} \in H_{0}^{2}(\omega),\left(\mathbf{u}_{I}, \dot{\eta}_{I}\right) \in L^{2}\left(\Omega_{\eta_{I}}\right) \times L^{2}(\omega)$ such that

$$
\begin{aligned}
& \min _{\bar{\omega}}\left(1+\eta_{I}\right)>0 \\
& \operatorname{div} \mathbf{u}_{I}=0 \text { in } \Omega_{\eta_{I}}, \\
& \mathbf{u}_{I} \cdot \mathbf{n}=0 \text { on } \Gamma_{0} \\
& \mathbf{u}_{I}\left(x, y, 1+\eta_{I}(x, y)\right) \cdot \mathbf{n}_{0}=\left(0,0, \dot{\eta}_{I}(x, y)\right)^{T} \cdot \mathbf{n}_{0} \text { on } \omega \\
& \int_{\omega} \dot{\eta}_{I}(x, y)=0
\end{aligned}
$$

where $\mathbf{n}_{0}$ denotes the unit normal to the initial position of the plate. We refer to [4] where one proves that the normal trace $\mathbf{u}_{I}\left(x, y, 1+\eta_{I}(x, y)\right) \cdot \mathbf{n}_{0},(x, y) \in \omega$ makes sense for $\mathbf{u}_{I} \in L^{2}\left(\Omega_{\eta_{I}}\right)$, with $\eta_{I} \in H_{0}^{2}(\omega)$. We shall say that $\left(\mathbf{u}_{\varepsilon}, \eta_{\varepsilon}\right)$ is a weak solution of the considered model on $(0, T)$ if it satisfies the following problem that will be denoted $\left(\mathcal{P}_{\varepsilon}\right)$ :

$-\mathbf{u}_{\varepsilon} \in V_{\eta_{\varepsilon}} \cap L^{\infty}\left(0, T ; L^{2}\left(\Omega_{\eta_{\varepsilon}}(t)\right)\right), \eta_{\varepsilon} \in W^{1, \infty}\left(0, T ; L^{2}(\omega)\right) \cap L^{\infty}\left(0, T ; H_{0}^{2}(\omega)\right)$,

- For $\varepsilon>0, \partial_{t} \eta_{\varepsilon} \in L^{2}\left(0, T ; H_{0}^{2}(\omega)\right)$,

- $\mathbf{u}_{\varepsilon}\left(t, x, y, 1+\eta_{\varepsilon}(t, x, y)\right)=\left(0,0, \partial_{t} \eta_{\varepsilon}(t, x, y)\right)^{T}$, for a. e. $(t, x, y) \in(0, T) \times$ $\omega$

- for all $(\phi, b) \in\left(V_{\eta_{\varepsilon}} \cap H^{1}\left(\widehat{\Omega}_{\eta_{\varepsilon}}\right)\right) \times\left(L^{2}\left(0, T ; H_{0}^{2}(\omega)\right) \times H^{1}\left(0, T ; L^{2}(\omega)\right)\right)$, such that $\phi\left(t, x, y, 1+\eta_{\varepsilon}(t, x, y)\right)=(0,0, b(t, x, y))^{T}$, for a. e. $(t, x, y) \in$ $(0, T) \times \omega$, we have for a. e. $t$ 


$$
\begin{gathered}
\int_{\Omega_{\eta_{\varepsilon}}(t)} \mathbf{u}_{\varepsilon}(t) \cdot \phi(t)-\int_{0}^{t} \int_{\Omega_{\eta_{\varepsilon}}(s)} \mathbf{u}_{\varepsilon} \cdot \partial_{t} \phi+\nu \int_{0}^{t} \int_{\Omega_{\eta_{\varepsilon}}(s)} \nabla \mathbf{u}_{\varepsilon}: \nabla \phi \\
+\int_{0}^{t} \int_{\Omega_{\eta_{\varepsilon}}(s)}\left(\mathbf{u}_{\varepsilon} \cdot \nabla\right) \mathbf{u}_{\varepsilon} \cdot \boldsymbol{\phi}-\int_{0}^{t} \int_{\omega}\left(\partial_{t} \eta_{\varepsilon}\right)^{2} b+\int_{\omega} \partial_{t} \eta_{\varepsilon}(t) b(t) \\
-\int_{0}^{t} \int_{\omega} \partial_{t} \eta_{\varepsilon} \partial_{t} b+\int_{0}^{t} \int_{\omega} \Delta \eta_{\varepsilon} \Delta b+\varepsilon \int_{0}^{t} \int_{\omega} \Delta \partial_{t} \eta_{\varepsilon} \Delta b \\
=\int_{0}^{t} \int_{\Omega_{\eta_{\varepsilon}}(t)} \mathbf{f} \cdot \boldsymbol{\phi}+\int_{0}^{t} \int_{\omega} g b+\int_{\Omega_{\eta_{I}}} \mathbf{u}_{I} \cdot \boldsymbol{\phi}(0)+\int_{\omega} \dot{\eta}_{I} b(0)
\end{gathered}
$$

In what follows, a solution of $\left(\mathcal{P}_{0}\right)$ will be denoted by $(\mathbf{u}, \eta)$ (instead of $\left.\left(\mathbf{u}_{0}, \eta_{0}\right)\right)$.

Remark 4 The test functions depends on the solution and thus, for $\varepsilon>0$, on $\varepsilon$.

Remark 5 The trace at time $t=0$ of $\phi \in H^{1}\left(\widehat{\Omega}_{\eta_{\varepsilon}}\right)$ such that $\phi(t, x, y, 1+$ $\left.\eta_{\varepsilon}(t, x, y)\right)=(0,0, b(t, x, y))^{T}$, for a. e. $(t, x, y) \in(0, T) \times \omega$, with $b \in H^{1}((0, T) \times$ $\omega)$ is well defined and makes sense at least in $L^{2}\left(\Omega_{\eta_{I}}\right)$. Indeed we can prove by density arguments $\left(C^{1}\left(\widehat{\Omega}_{\eta_{\varepsilon}}\right)\right.$ is dense in $H^{1}\left(\widehat{\Omega}_{\eta_{\varepsilon}}\right)$ since the domain is a continuous subgraph, see for instance [1], Thm 2, p. 54) that

$-\int_{\Omega_{\eta_{\varepsilon}(0)}}|\phi(0)|^{2}=2 \int_{0}^{T} \int_{\Omega_{\eta_{\varepsilon}}(t)} \psi \phi \partial_{t} \phi+\int_{0}^{T} \int_{\Omega_{\eta_{\varepsilon}}(t)}|\phi|^{2} \partial_{t} \psi+\int_{0}^{T} \int_{\omega}|b|^{2} \psi \partial_{t} \eta_{\varepsilon}$ where $\psi$ belongs to $\mathcal{D}([0, T))$ and satisfies $\psi(0)=1$. The right hand side of this equality makes sense for any $\phi \in H^{1}\left(\widehat{\Omega}_{\eta_{\varepsilon}}\right)$ such that $\phi\left(t, x, y, 1+\eta_{\varepsilon}(t, x, y)\right)=$ $(0,0, b(t, x, y))^{T}$, for a. e. $(t, x, y) \in(0, T) \times \omega$, with $b \in H^{1}((0, T) \times \omega)$, since $\partial_{t} \eta_{\varepsilon} \in L^{\infty}\left(0, T ; L^{2}(\omega)\right)$ and $b \in L^{4}((0, T) \times \omega)$.

\section{Main Result}

We make the following hypotheses on the data (bulk forces and initial data):

$$
\begin{gathered}
\mathbf{f} \in L_{l o c}^{2}\left((0,+\infty) \times \mathbb{R}^{2}\right), g \in L_{l o c}^{2}((0,+\infty) \times \omega), \\
\mathbf{u}_{I} \in L^{2}\left(\Omega_{\eta_{I}}\right), \dot{\eta}_{I} \in L^{2}(\omega), \eta_{I} \in H_{0}^{2}(\omega),
\end{gathered}
$$

and we assume moreover that conditions (21) are satisfied.

First we recall that, for $\varepsilon>0$, there exists at least one weak solution provided that the plate does not touch the bottom of the fluid cavity, in other words as long as $\min _{(x, y) \in \bar{\omega}}\left(1+\eta_{\varepsilon}(t, x, y)\right)>0$. The proof of the following theorem can be found in [4].

Theorem 1 Let $\varepsilon$ be strictly positive. Under assumptions (21), (23), and if $\min _{(x, y) \in \bar{\omega}} 1+\eta_{I}(x, y)>0$, there exists $T_{\varepsilon}^{*} \in(0,+\infty]$ and a weak solution 
$\left(\mathbf{u}_{\varepsilon}, \eta_{\varepsilon}\right)$ of $\left(\mathcal{P}_{\varepsilon}\right)$ on $[0, T], T<T_{\varepsilon}^{*}$. This solution satisfies an energy estimate for all $T<T_{\varepsilon}^{*}$ :

$$
\begin{gathered}
\left\|\mathbf{u}_{\varepsilon}\right\|_{L^{\infty}\left(0, T ; L^{2}\left(\Omega_{\eta_{\varepsilon}}(t)\right)\right)}+\left\|\nabla \mathbf{u}_{\varepsilon}\right\|_{L^{2}\left(0, T ; L^{2}\left(\Omega_{\eta_{\varepsilon}}(t)\right)\right)} \\
+\left\|\partial_{t} \eta_{\varepsilon}\right\|_{L^{\infty}\left(0, T ; L^{2}(\omega)\right)}+\left\|\Delta \eta_{\varepsilon}\right\|_{L^{\infty}\left(0, T ; L^{2}(\omega)\right)}+\sqrt{\varepsilon}\left\|\Delta \partial_{t} \eta_{\varepsilon}\right\|_{L^{2}\left(0, T ; L^{2}(\omega)\right)} \\
\leq C\left(T,\left\|\mathbf{u}_{I}\right\|_{L^{2}\left(\Omega_{\eta_{I}}\right)},\|\mathbf{f}\|_{L^{2}\left((0, T) \times \mathbb{R}^{2}\right)},\|g\|_{L^{2}((0, T) \times \omega)},\left\|\eta_{I}\right\|_{H_{0}^{2}(\omega)},\left\|\dot{\eta}_{I}\right\|_{L^{2}(\omega)}\right),
\end{gathered}
$$

where $C>0$ is nondecreasing with respect to its arguments. Moreover, we have the following alternative

- either $T_{\varepsilon}^{*}=+\infty$,

- or $\lim _{t \rightarrow T_{\varepsilon}^{*}} \min _{\bar{\omega}}\left(1+\eta_{\varepsilon}\right)=0$.

Since $\mathbf{u}_{\varepsilon}$ is bounded in $L^{2}\left(0, T ; H^{1}\left(\Omega_{\eta_{\varepsilon}}(t)\right)\right)$ uniformly in $\varepsilon$ and thanks to the regularity of the moving elastic boundary, the trace $\gamma_{\eta_{\varepsilon}(t)}\left(\mathbf{u}_{\varepsilon}\right)$ is bounded in $L^{2}\left(0, T ; W^{1-1 / p, p}(\omega)\right), \forall 1<p<2$ and in $L^{2}\left(0, T ; H^{s}(\omega)\right), \forall 0 \leq s<\frac{1}{2}$ uniformly in $\varepsilon$ (see Lemma 2 and (14)). Thus, thanks to the equality of the velocities (4),

$\partial_{t} \eta_{\varepsilon}$ is bounded, uniformly in $\varepsilon$, in $L^{2}\left(0, T ; W^{1-1 / p, p}(\omega)\right), \forall 1<p<2$,

and

$\partial_{t} \eta_{\varepsilon}$ is bounded, uniformly in $\varepsilon$, in $L^{2}\left(0, T ; H^{s}(\omega)\right), \forall 0 \leq s<\frac{1}{2}$.

This will be one of the key argument for the derivation of the compactness properties of the sequence $\left(\mathbf{u}_{\varepsilon}, \eta_{\varepsilon}\right)$. In particular thanks to (25) we can control, uniformly in $\varepsilon$, the space high frequencies of $\partial_{t} \eta_{\varepsilon}$ in $L^{2}\left(0, T ; L^{2}(\omega)\right)$.

In all that follows, the characteristic function of $\widehat{\Omega}_{\eta_{\varepsilon}}$ will be denoted by $\rho_{\varepsilon}$ and $\rho_{\varepsilon} v$ will denote the function equal to $v$ in $\widehat{\Omega}_{\eta_{\varepsilon}}$ and zero elsewhere. Moreover, we choose $M$ large enough such that for all $\varepsilon>0,1+\eta_{\varepsilon}(t, x, y) \leq M, \forall(t, x, y) \in$ $[0, T] \times \bar{\omega}$, which is made possible by (12) and (13). We set

$$
\overline{\mathbf{u}}_{\varepsilon}=\mid \begin{array}{ll}
\mathbf{u}_{\varepsilon} & \text { in } \widehat{\Omega}_{\eta_{\varepsilon}} \\
\left(0,0, \partial_{t} \eta_{\varepsilon}\right)^{T} & \text { in } \widehat{\mathcal{C}_{M}} \backslash \bar{\Omega}_{\eta_{\varepsilon}} .
\end{array}
$$

In all that follows, for any $\mathbf{v}$ in $L^{2}\left(0, T ; H_{0, \Gamma_{0}}^{1}\left(\Omega_{\eta_{\varepsilon}}(t)\right)\right)$, such that $\gamma_{\delta(t)}(\mathbf{v})=$ $(0,0, b)^{T}, b \in L^{2}\left(0, T ; H_{0}^{1}(\omega)\right), \overline{\mathbf{v}}$ is defined by $(15)$.

The main results of the present paper are

Proposition 1 The sequence $\left(T_{\varepsilon}^{*}\right)_{\varepsilon>0}$ is bounded from below away from zero and the following convergences (up to the extractions of subsequences) hold as $\varepsilon$ 
goes to zero:

$$
\begin{array}{lllll}
\eta_{\varepsilon} & \rightarrow & \text { strongly in } & C^{0}\left([0, T] ; C^{0}(\bar{\omega})\right), \\
\eta_{\varepsilon} & \rightarrow & \text { weakly in } & L^{2}\left(0, T ; H_{0}^{2}(\omega)\right), \\
\partial_{t} \eta_{\varepsilon} & \rightarrow & \partial_{t} \eta & \text { strongly in } & L^{2}\left(0, T ; L^{2}(\omega)\right), \\
\rho_{\varepsilon} \mathbf{u}_{\varepsilon} & \rightarrow & \rho \mathbf{u} & \text { strongly in } & L^{2}\left(0, T ; L^{2}\left(\mathcal{C}_{M}\right)\right), \\
\overline{\mathbf{u}}_{\varepsilon} & \rightarrow \overline{\mathbf{u}} & \text { strongly in } & L^{2}\left(0, T ; L^{2}\left(\mathcal{C}_{M}\right)\right), \\
\rho_{\varepsilon} \nabla \mathbf{u}_{\varepsilon} & \rightarrow \rho \nabla \mathbf{u} & \text { weakly in } & L^{2}\left(0, T ; L^{2}\left(\mathcal{C}_{M}\right)\right),
\end{array}
$$

where $T>0$ is a lower bound of $T_{\varepsilon}^{*}$ independent of $\varepsilon$. Moreover

$$
\gamma_{\eta(t)}(\mathbf{u})=\left(0,0, \partial_{t} \eta\right)^{T}
$$

This enables us to pass to the limit in the equation (22) as $\varepsilon$ tends to zero and thus obtain the

Theorem 2 Under assumptions (21), (23), and if $\min _{(x, y) \in \bar{\omega}} 1+\eta_{I}(x, y)>0$, there exists $T^{*} \in(0,+\infty]$ and a weak solution $(\mathbf{u}, \eta)$ of $\left(\mathcal{P}_{0}\right)$ on $[0, T], T<T^{*}$. This solution satisfies energy estimates for all $T<T^{*}$ :

$$
\begin{gathered}
\|\mathbf{u}\|_{L^{\infty}\left(0, T ; L^{2}\left(\Omega_{\eta}(t)\right)\right)}+\|\nabla \mathbf{u}\|_{L^{2}\left(0, T ; L^{2}\left(\Omega_{\eta}(t)\right)\right)} \\
+\left\|\partial_{t} \eta\right\|_{L^{\infty}\left(0, T ; L^{2}(\omega)\right)}+\|\eta\|_{L^{\infty}\left(0, T ; H_{0}^{2} \omega\right)} \\
\leq C\left(T,\left\|\mathbf{u}_{I}\right\|_{L^{2}\left(\Omega_{\eta_{I}}\right)},\|\mathbf{f}\|_{L^{2}\left((0, T) \times \mathbb{R}^{2}\right)},\|g\|_{L^{2}((0, T) \times \omega)},\left\|\eta_{I}\right\|_{H_{0}^{2}(\omega)},\left\|\dot{\eta}_{I}\right\|_{L^{2}(\omega)}\right),
\end{gathered}
$$

where $C>0$ is nondecreasing with respect to its arguments. The following alternatives are satisfied

- either $T^{*}=+\infty$,

- or $\lim _{t \rightarrow T^{*}} \min _{\bar{\omega}}(1+\eta)=0$.

\section{Proof of Proposition 1}

First we prove that Proposition 1 holds true. We have to verify that $T_{\varepsilon}^{*}$ is bounded from below independently of $\varepsilon$, and obtain compactness properties on $\left(\mathbf{u}_{\varepsilon}, \partial_{t} \eta_{\varepsilon}\right)$ in order to prove the desired strong convergences that will enable us to pass to the limit in $\left(\mathcal{P}_{\varepsilon}\right)$ as $\varepsilon$ goes to zero.

\section{- Lower bound of $T_{\varepsilon}^{*}$.}

For $\varepsilon>0$ the solution $\eta_{\varepsilon}$ is bounded uniformly in $\varepsilon$ in $L^{\infty}\left(0, T ; H_{0}^{2}(\omega)\right) \cap$ $W^{1, \infty}\left(0, T ; L^{2}(\omega)\right)$ for all $T<T_{\varepsilon}^{*}$. Thus from $(13), \eta_{\varepsilon}$ is bounded uniformly in $\varepsilon$ in $C^{0, \mu}\left([0, T] ; C^{0}(\bar{\omega})\right), 0<\mu<\frac{1}{2}$. Consequently

$$
1+\eta_{\varepsilon}(t, x, y) \geq\left(1+\eta_{I}(x, y)\right)-C t^{\mu}, \quad \forall(t, x, y) \in\left[0, T_{\varepsilon}^{*}\right) \times \bar{\omega}
$$


where $C$ depends only on the data of the problem. Thus $T_{\varepsilon}^{*}$ is bounded from below by a time independent of $\varepsilon$. Let $T$ be such that

$$
\forall \varepsilon>0, \min _{(t, x, y) \in[0, T] \times \bar{\omega}}\left(1+\eta_{\varepsilon}(t, x, y)\right) \geq \alpha>0,
$$

where $\alpha$ is chosen such that $\min _{(x, y) \in \bar{\omega}}\left(1+\eta_{I}(x, y)\right) \geq 2 \alpha>0$.

- Convergences of the sequence $\left(\mathbf{u}_{\varepsilon}, \eta_{\varepsilon}\right)$.

From (12) and the compact injection (13) we deduce easily the first two convergences announced in Proposition 1. Next we prove strong convergence properties for the fluid and the structure velocities. The solution $\left(\mathbf{u}_{\varepsilon}, \eta_{\varepsilon}\right)_{\varepsilon>0}$ we build verifies estimate (9) and (25). Furthermore, since $\mathbf{u}_{\varepsilon}$ is bounded uniformly in $\varepsilon$ in $L^{2}\left(0, T ; H^{1}\left(\Omega_{\eta_{\varepsilon}}(t)\right)\right)$, it is easy to verify that $\mathbf{w}_{\varepsilon}$, defined by $\mathbf{w}_{\varepsilon}(t, x, y, z)=$ $\mathbf{u}_{\varepsilon}\left(t, x, y, z\left(1+\eta_{\varepsilon}(t, x, y)\right)\right.$, is bounded uniformly in $\varepsilon$ in $L^{2}\left(0, T ; W^{1, p}\left(\mathcal{C}_{1}\right)\right), \forall 1<$ $p<2$. This implies, thanks to Sobolev injections (see [1], Th. 7.58, p. 218) that $\mathbf{w}_{\varepsilon}$ is uniformly bounded in $L^{2}\left(0, T ; H^{\theta}\left(\mathcal{C}_{1}\right)\right)$, for any $\theta<1$. Moreover $\partial_{t} \eta_{\varepsilon}$ is uniformly bounded in $L^{2}\left(0, T ; H^{s}(\omega)\right), \forall 0 \leq s<\frac{1}{2}$. Consequently, $\mathbf{w}_{\varepsilon}-\mathcal{R}_{\alpha}\left(\partial_{t} \eta_{\varepsilon}\right)$ is uniformly bounded in $L^{2}\left(0, T ; H^{s}\left(\mathcal{C}_{1}\right)\right)$, for any $0 \leq s<\frac{1}{2}$ and its extension by zero for $z \geq 1$ is uniformly bounded in $L^{2}\left(0, T ; H^{s}\left(\mathcal{C}_{L}\right)\right)$, for any $s<\frac{1}{2}, L \geq 1$ (see [19]). Thus if we extend $\mathbf{w}_{\varepsilon}$ by $\left(0,0, \partial_{t} \eta_{\varepsilon}\right)^{T}$ for $z \geq 1$, this extention is uniformly bounded in $L^{2}\left(0, T ; H^{s}\left(\mathcal{C}_{L}\right)\right), \forall 0 \leq s<\frac{1}{2}, L \geq 1$. Consequently, since the change of variables $\phi_{\varepsilon}(t, x, y, z)=\left(x, y, z\left(1+\eta_{\varepsilon}(t, x, y)\right)^{T}\right.$ is in $L^{\infty}\left(0, T ; C^{0, \beta}\left(\mathcal{C}_{L}\right)\right), \forall \beta<1$ as well as its inverse, it is easy to verify that

$\overline{\mathbf{u}}_{\varepsilon}$ is bounded, uniformly in $\varepsilon$, in $L^{2}\left(0, T ; H^{s^{\prime}}\left(\mathcal{C}_{M}\right)\right), \forall 0 \leq s^{\prime}<s, \forall s<\frac{1}{2}$.

Moreover thanks to the Sobolev injections, $\mathbf{w}_{\varepsilon}$ is bounded uniformly in $\varepsilon$ in $L^{2}\left(0, T ; L^{q}\left(\mathcal{C}_{1}\right)\right), \forall q<6$ and $\partial_{t} \eta_{\varepsilon}$ is uniformly bounded in $L^{2}\left(0, T ; L^{r}(\omega)\right), \forall r<$ 4 , and thus

$\overline{\mathbf{u}}_{\varepsilon}$ is bounded, uniformly in $\varepsilon$, in $L^{2}\left(0, T ; L^{r}\left(\mathcal{C}_{M}\right)\right), \forall r<4$.

Nevertheless, these bounds are not sufficient to obtain the desired strong convergences. We are going to use the following Lemma that characterizes the compact sets of $L^{p}(0, T ; X)$ where $X$ is a Banach space (see [23]).

Lemma 9 Let $X$ be a Banach space and $F \hookrightarrow L^{q}(0, T ; X)$ with $1 \leq q<\infty$. Then $F$ is a relativelly compact set of $L^{q}(0, T ; X)$ if and only if

i) $\left\{\int_{t_{1}}^{t_{2}} f(t) d t, f \in F\right\}$ is relativelly compact in $X, \forall 0<t_{1}<t_{2}<T$

ii) $\|f(t+h)-f(t)\|_{L^{q}(0, T ; X)} \longrightarrow 0$ as $h$ goes to zero, uniformly with respect to $f$ in $F$.

We are going to apply Lemma 9 to $F=\left(\overline{\mathbf{u}}_{\varepsilon}, \partial_{t} \eta_{\varepsilon}\right)_{\varepsilon>0}, q=2$ and $X=$ $L^{2}\left(\mathcal{C}_{M}\right) \times L^{2}(\omega)$. The first point $\left.i\right)$ is clearly satisfied thanks to $(25),(28)$ and we 
have to verify the second point. Given any $h>0$, we denote $g^{-}(t, \cdot)=g(t-h, \cdot)$ and $g^{+}(t, \cdot)=g(t+h, \cdot)$. The assertion $\left.i i\right)$ is a consequence of the following Lemma:

Lemma 10 Let $T>0$ such that $\min _{[0, T] \times \bar{\omega}}\left(1+\eta_{\varepsilon}\right) \geq \alpha>0$. We have $\forall \beta>$ $0, \exists h_{0}>0$, s. $t . \forall \varepsilon>0, \forall h \leq h_{0}$

$$
\int_{0}^{T} \int_{\mathcal{C}_{M}} \rho_{\varepsilon}\left|\overline{\mathbf{u}}_{\varepsilon}-\overline{\mathbf{u}}_{\varepsilon}^{-}\right|^{2}+\int_{0}^{T} \int_{\omega}\left(\partial_{t} \eta_{\varepsilon}-\partial_{t} \eta_{\varepsilon}^{-}\right)^{2} \leq \beta
$$

and

$$
\int_{0}^{T} \int_{\mathcal{C}_{M}}\left|\rho_{\varepsilon} \overline{\mathbf{u}}_{\varepsilon}-\rho_{\varepsilon}^{-} \overline{\mathbf{u}}_{\varepsilon}^{-}\right|^{2} \leq \beta,
$$

with $\eta_{\varepsilon}$ extended by $\eta_{I}$ for $t<0$ and $\overline{\mathbf{u}}_{\varepsilon}$ and $\partial_{t} \eta_{\varepsilon}$ extended by 0 for $t<0$, and where $\rho_{\varepsilon}$ denotes the characteristic function of $\widehat{\Omega}_{\eta_{\varepsilon}}$.

Proof:

We first show that (30) implies (31). Indeed:

$$
\left|\rho_{\varepsilon} \overline{\mathbf{u}}_{\varepsilon}-\rho_{\varepsilon}^{-} \overline{\mathbf{u}}_{\varepsilon}^{-}\right|^{2} \leq C\left(\rho_{\varepsilon}\left|\overline{\mathbf{u}}_{\varepsilon}-\overline{\mathbf{u}}_{\varepsilon}^{-}\right|^{2}+\left|\rho_{\varepsilon}-\rho_{\varepsilon}^{-}\right|\left|\overline{\mathbf{u}}_{\varepsilon}^{-}\right|^{2}\right) .
$$

The estimate of the first contribution comes from (30). For the second contribution we use the fact that $\overline{\mathbf{u}}_{\varepsilon}$ is bounded uniformly in $\varepsilon$ in $L^{2}\left(0, T ; L^{3}\left(\mathcal{C}_{M}\right)\right)$ (see (29)).

$\left|\int_{0}^{T} \int_{\mathcal{C}_{M}}\right| \rho_{\varepsilon}-\left.\rho_{\varepsilon}^{-}|| \overline{\mathbf{u}}_{\varepsilon}\right|^{2} \mid \leq \int_{0}^{T}\left\|\rho_{\varepsilon}-\rho_{\varepsilon}^{-}\right\|_{L^{3}\left(\mathcal{C}_{M}\right)}\left\|\overline{\mathbf{u}}_{\varepsilon}\right\|_{L^{3}\left(\mathcal{C}_{M}\right)}^{2} \leq C \int_{0}^{T}\left\|\rho_{\varepsilon}-\rho_{\varepsilon}^{-}\right\|_{L^{3}\left(\mathcal{C}_{M}\right)}$.

Remember now that $\partial_{t} \eta_{\varepsilon}$ is bounded in $L^{\infty}\left(0, T ; L^{2}(\omega)\right)$ uniformly in $\varepsilon$, thus

$$
\begin{aligned}
\int_{\mathcal{C}_{M}}\left|\rho_{\varepsilon}-\rho_{\varepsilon}^{-}\right|^{3} & =\int_{\omega}\left|\eta_{\varepsilon}-\left(\eta_{\varepsilon}\right)^{-}\right| \\
& =\int_{\omega}\left|\int_{t-h}^{t} \partial_{t} \eta_{\varepsilon}(s) d s\right| \\
& \leq \int_{\omega} \int_{t-h}^{t}\left|\partial_{t} \eta_{\varepsilon}(s)\right| d s \\
& \leq C h .
\end{aligned}
$$

It leads to

$$
\left.\left|\int_{0}^{T} \int_{\mathcal{C}_{M}}\left(\rho_{\varepsilon}-\rho_{\varepsilon}^{-}\right)\right| \overline{\mathbf{u}}_{\varepsilon}\right|^{2} \mid \leq C h^{\frac{1}{3}} .
$$

This shows (30) implies (31). 
To prove (30) we are going to make a suitable choice for the test functions in the weak formulation satisfied by $\mathbf{u}_{\varepsilon}$ and $\eta_{\varepsilon}$ :

$$
\begin{aligned}
& \int_{\Omega_{\eta_{\varepsilon}}(T)} \mathbf{u}_{\varepsilon}(T) \cdot \phi(T)-\int_{0}^{T} \int_{\Omega_{\eta_{\varepsilon}}(t)} \mathbf{u}_{\varepsilon} \cdot \partial_{t} \phi+\nu \int_{0}^{T} \int_{\Omega_{\eta_{\varepsilon}}(t)} \nabla \mathbf{u}_{\varepsilon}: \nabla \phi \\
& +\int_{0}^{T} \int_{\Omega_{\eta_{\varepsilon}}(t)}\left(\mathbf{u}_{\varepsilon} \cdot \nabla\right) \mathbf{u}_{\varepsilon} \cdot \phi-\int_{0}^{T} \int_{\omega}\left(\partial_{t} \eta_{\varepsilon}\right)^{2} b+\int_{\omega} \partial_{t} \eta_{\varepsilon}(T) b(T) \\
& -\int_{0}^{T} \int_{\omega} \partial_{t} \eta_{\varepsilon} \partial_{t} b+\int_{0}^{T} \int_{\omega} \Delta \eta_{\varepsilon} \Delta b+\varepsilon \int_{0}^{T} \int_{\omega} \Delta \partial_{t} \eta_{\varepsilon} \Delta b \\
& =\int_{0}^{T} \int_{\Omega_{\eta_{\varepsilon}}(t)} \mathbf{f} \cdot \boldsymbol{\phi}+\int_{0}^{T} \int_{\omega} g b+\int_{\Omega_{\eta_{I}}} \mathbf{u}_{I} \cdot \boldsymbol{\phi}(0)+\int_{\omega} \dot{\eta}_{I} b(0),
\end{aligned}
$$

for all $(\phi, b) \in\left(V_{\eta_{\varepsilon}} \cap H^{1}\left(\widehat{\Omega}_{\eta_{\varepsilon}}\right)\right) \times\left(L^{2}\left(0, T ; H_{0}^{2}(\omega)\right) \cap H^{1}\left(0, T ; L^{2}(\omega)\right)\right.$,

s. t. $\phi\left(t, x, y, 1+\eta_{\varepsilon}(t, x, y)\right)=(0,0, b(t, x, y))^{T}$, for a. e. $(t, x, y) \in(0, T) \times \omega$.

We are going to study separately the low frequencies and the high frequencies of $\partial_{t} \eta_{\varepsilon}$ and take advantage of the fact that $\partial_{t} \eta_{\varepsilon}$ is bounded in $L^{2}\left(0, T ; H^{s}(\omega)\right)$, $\forall 0 \leq s<\frac{1}{2}$ uniformly in $\varepsilon$ (see (25)). This implies that we can control, uniformly in $\varepsilon$, the space high frequencies of $\partial_{t} \eta_{\varepsilon}$ in $L^{2}\left(0, T ; L^{2}(\omega)\right)$.

\section{- Definition of admissible test functions}

First we introduce a basis of $H_{0}^{2}(\omega) \cap L_{0}^{2}(\omega)$ by taking eigenfunctions $\left(\xi_{i}\right)_{i \in \mathbb{N}}$ defined by:

$$
\left\{\begin{array}{l}
\int_{\omega} \Delta \xi_{i} \Delta b=\lambda_{i} \int_{\omega} \xi_{i} b, \quad \forall b \in H_{0}^{2}(\omega) \text { s.t. } \int_{\omega} b=0, \\
\xi_{i} \in H_{0}^{2}(\omega), \int_{\omega} \xi_{i}=0,
\end{array}\right.
$$

with $\left(\lambda_{i}\right)_{i \in \mathbb{N}}$ the sequence of increasing eigenvalues: $\lambda_{i}>0, \lambda_{i} \rightarrow+\infty$. We choose $\left(\xi_{i}\right)_{i \in \mathbb{N}}$ orthonormal in $L^{2}(\omega)$. We denote by $d^{N_{0}}$ the $L^{2}$-projection on the finite dimensional space $\operatorname{span}\left(\xi_{i}\right)_{0 \leq i \leq N_{0}}$ of any function $d$ and by $d^{h f, N_{0}}$ the difference $d-d^{N_{0}}$. Thanks to the choice of the $\xi_{i}$ the $L^{2}{ }^{2}$-projection on the finite dimensional space $\operatorname{span}\left(\xi_{i}\right)_{0 \leq i \leq N_{0}}$ is stable in the $L^{2}{ }^{-}$norm as well as in the $H_{0}^{2}$-norm. In what follows, we will use the following property, obtained by Hilbertian interpolation, holds true:

$$
\forall d \in H^{s}(\omega), 0 \leq s<\frac{1}{2}, \quad\left\|d^{h f, N_{0}}\right\|_{L^{2}(\omega)} \leq \lambda_{N_{0}}^{-\frac{s}{2}}\|d\|_{H^{s}(\omega)}, .
$$

Next, for $\sigma>1$ we define $\mathbf{v}_{\sigma}$ by

$$
\mathbf{v}_{\sigma}(x, y, z)=\left(\sigma v_{1}(x, y, \sigma z), \sigma v_{2}(x, y, \sigma z), v_{3}(x, y, \sigma z)\right) .
$$

If $\mathbf{v}$ is divergence free $\mathbf{v}_{\sigma}$ is also divergence free.

We want now to define admissible test functions. We set

$$
b_{\varepsilon}=\int_{t-h}^{t} \partial_{t} \eta_{\varepsilon}^{N_{0}}(s) d s
$$


and

$$
\phi_{\varepsilon}=\int_{t-h}^{t}\left(\overline{\left(\mathbf{u}_{\varepsilon}-\mathcal{R}_{\alpha}\left(\partial_{t} \eta_{\varepsilon}\right)\right)^{\lambda}}\right)_{\sigma}(s) d s+\int_{t-h}^{t} \overline{\mathcal{R}_{\alpha}\left(\partial_{t} \eta_{\varepsilon}^{N_{0}}\right)}(s) d s
$$

where the extension $\mathbf{v} \mapsto \overline{\mathbf{v}}$ is defined by (15) and where $\mathcal{R}_{\alpha}$ is the lifting operator defined at Lemma 6. Moreover a space regularization of $\mathbf{v}_{\varepsilon}=\mathbf{u}_{\varepsilon}-\mathcal{R}_{\alpha}\left(\partial_{t} \eta_{\varepsilon}\right)$, denoted by $\mathbf{v}_{\varepsilon}^{\lambda}$ has been introduced in order to have $\phi_{\varepsilon}$ uniformly bounded in $H^{1}\left(0, T ; H^{1}\left(\mathcal{C}_{M}\right)\right)$. It verifies $\operatorname{div}\left(\mathbf{v}_{\varepsilon}^{\lambda}\right)=0, \mathbf{v}_{\varepsilon}^{\lambda} \in L^{2}\left(0, T ; H_{0}^{1}\left(\Omega_{\eta_{\varepsilon}}(t)\right)\right)$ and

$$
\begin{aligned}
& \left\|\mathbf{v}_{\varepsilon}-\mathbf{v}_{\varepsilon}^{\lambda}\right\|_{L^{2}\left(0, T ; L^{2}\left(\Omega_{\eta_{\varepsilon}}(t)\right)\right)} \longrightarrow 0, \text { uniformly in } \varepsilon \text {, as } \lambda \text { goes to zero, } \\
& \left\|\mathbf{v}_{\varepsilon}^{\lambda}\right\|_{L^{2}\left(0, T ; H^{1}\left(\Omega_{\eta_{\varepsilon}}(t)\right)\right)} \leq C_{\lambda} .
\end{aligned}
$$

Note that the construction of $\mathbf{v}_{\varepsilon}^{\lambda}$ relies on the fact that $\eta_{\varepsilon}$ converges uniformly to $\eta$ and that the plate does not touch the bottom of the fluid cavity. Moreover, the uniform convergence of $\left(\mathbf{v}_{\varepsilon}^{\lambda}\right)_{\lambda}$ as $\lambda \longrightarrow 0$ in $L^{2}\left(0, T ; L^{2}\left(\Omega_{\eta_{\varepsilon}}(t)\right)\right)$ is made possible since $\overline{\mathbf{v}}_{\varepsilon}$ is uniformly bounded in $L^{2}\left(0, T ; H^{s^{\prime}}\left(\mathcal{C}_{M}\right)\right), 0<s^{\prime}<1 / 2$ thanks to (17), (25) and (28). Note that, with this choice,

$$
\begin{gathered}
\left\|\partial_{t} \eta_{\varepsilon}^{N_{0}}\right\|_{L^{\infty}\left(0, T ; L^{2}(\omega)\right)} \leq C, \quad\left\|\partial_{t} \eta_{\varepsilon}^{N_{0}}\right\|_{L^{2}\left(0, T ; H^{s}(\omega)\right)} \leq C, \\
\left\|b_{\varepsilon}\right\|_{W^{1, \infty}\left(0, T ; L^{2}(\omega)\right)} \leq C, \quad\left\|b_{\varepsilon}\right\|_{H^{1}\left(0, T ; H^{s}(\omega)\right)} \leq C, \\
\left\|\overline{\mathbf{v}_{\varepsilon}^{\lambda}}\right\|_{L^{\infty}\left(0, T ; L^{2}\left(\mathcal{C}_{M}\right)\right)} \leq C,\left\|\overline{\mathbf{v}_{\varepsilon}^{\lambda}}\right\|_{L^{2}\left(0, T ; H^{s^{\prime}}\left(\left(\mathcal{C}_{M}\right)\right)\right.} \leq C, \forall s^{\prime}<s<1 / 2,
\end{gathered}
$$

and

$\left\|\partial_{t} \eta_{\varepsilon}^{N_{0}}\right\|_{L^{\infty}\left(0, T ; H^{2}(\omega)\right)} \leq C_{N_{0}},\left\|b_{\varepsilon}\right\|_{W^{1, \infty}\left(0, T ; H^{2}(\omega)\right)} \leq C_{N_{0}},\left\|\overline{\mathbf{v}_{\varepsilon}^{\lambda}}\right\|_{L^{2}\left(0, T ; H^{1}\left(\mathcal{C}_{M}\right)\right)} \leq C_{\lambda}$,

where $C$ denotes and will denote in all that follows a strictly positive constant that depends only on the data and not on $\varepsilon$ and $N_{0}$, and $C_{N_{0}}$ (resp. $C_{\lambda}$ ) denotes and will denote a strictly positive constant that depends on the data and not on $\varepsilon$ but may depend on $N_{0}$ (resp. $\lambda$ ). The integer $N_{0}$ (resp. the real $\lambda$ ) will be fixed later on and will be large enough (resp. small enough). Then for well chosen $\sigma,\left(\phi_{\varepsilon}, b_{\varepsilon}\right)$ are admissible tests functions. Indeed, $\phi_{\varepsilon}$ is divergence free thanks to the definitions of the lifting operator $\mathcal{R}_{\alpha}$, the extension operator $\mathbf{v} \mapsto \overline{\mathbf{v}}$, the operator $\mathbf{v} \mapsto \mathbf{v}_{\sigma}$ and the definition of the regularization $\mathbf{v} \mapsto \mathbf{v}^{\lambda}$. Moreover $\phi_{\varepsilon}$ belongs to $H^{1}\left(0, T ; H^{1}\left(\mathcal{C}_{M}\right)\right)$. The function $b_{\varepsilon}$ belongs to $H^{1}\left(0, T ; H_{0}^{2}(\omega)\right)$. Both of them are bounded in the previous spaces independently of $\varepsilon$ but not of $N_{0}$ and $\lambda$. Moreover since

$$
\left\|\eta_{\varepsilon}\right\|_{L^{\infty}\left(0, T ; H_{0}^{2}(\omega)\right) \cap W^{1, \infty}\left(0, T ; L^{2}(\omega)\right)} \leq C,
$$

and remembering the imbedding (13), we have

$$
\left\|\eta_{\varepsilon}-\eta_{\varepsilon}^{-}\right\|_{C^{0}([0, T] \times \bar{\omega})} \leq C h^{\mu}, \quad 0<\mu<\frac{1}{2} .
$$


Thus if $\sigma$ is such that $\sigma \geq 1+\frac{2 C}{\alpha} h^{\mu}$, we have

$$
\phi_{\varepsilon}\left(t, x, y, 1+\eta_{\varepsilon}(t, x, y)\right)=\left(0, \int_{t-h}^{t} \partial_{t} \eta_{\varepsilon}^{N_{0}}(s, x, y) d s\right)^{T}, \text { on } \omega \text {. }
$$

In the sequel we choose $\sigma=1+\frac{2 C}{\alpha} h^{\mu}, 0<\mu<\frac{1}{2}$. Hence, with the choice of test functions we made, (33) writes:

$$
\begin{aligned}
& \left.\left.-\int_{0}^{T} \int_{\Omega_{\eta_{\varepsilon}}(t)} \mathbf{u}_{\varepsilon} \cdot\left(\left(\overline{\mathbf{v}_{\varepsilon}^{\lambda}}\right)_{\sigma}-\left(\overline{\mathbf{v}_{\varepsilon}^{\lambda}}\right)_{\sigma}^{-}\right) \int_{0}^{T} \int_{\Omega_{\eta_{\varepsilon}}(t)} \mathbf{u}_{\varepsilon} \cdot\left(\overline{\mathcal{R}_{\alpha}\left(\partial_{t} \eta_{\varepsilon}^{N_{0}}\right.}\right)-\overline{\mathcal{R}_{\alpha}\left(\partial_{t} \eta_{\varepsilon}^{N_{0}}\right.}\right)^{-}\right) \\
& +\int_{0}^{T} \int_{\Omega_{\eta_{\varepsilon}}(t)}\left(\mathbf{u}_{\varepsilon} \cdot \nabla\right) \mathbf{u}_{\varepsilon} \cdot \phi_{\varepsilon}+\nu \int_{0}^{T} \int_{\Omega_{\eta_{\varepsilon}}(t)} \nabla \mathbf{u}_{\varepsilon}: \nabla \phi_{\varepsilon} \\
& +\int_{\Omega_{\eta_{\varepsilon}}(T)} \mathbf{u}_{\varepsilon}(T) \cdot \phi_{\varepsilon}(T)-\int_{0}^{T} \int_{\omega} \partial_{t} \eta_{\varepsilon} \partial_{t}\left(\eta_{\varepsilon}^{N_{0}}-\left(\eta_{\varepsilon}^{N_{0}}\right)^{-}\right) \\
& -\int_{0}^{T} \int_{\omega}\left(\partial_{t} \eta_{\varepsilon}\right)^{2}\left(\eta_{\varepsilon}^{N_{0}}-\left(\eta_{\varepsilon}^{N_{0}}\right)^{-}\right)+\int_{0}^{T} \int_{\omega} \Delta \eta_{\varepsilon} \Delta\left(\eta_{\varepsilon}^{N_{0}}-\left(\eta_{\varepsilon}^{N_{0}}\right)^{-}\right) \\
& +\varepsilon \int_{0}^{T} \int_{\omega} \Delta \partial_{t} \eta_{\varepsilon} \Delta\left(\eta_{\varepsilon}^{N_{0}}-\left(\eta_{\varepsilon}^{N_{0}}\right)^{-}\right)+\int_{\omega} \partial_{t} \eta_{\varepsilon}(T)\left(\eta_{\varepsilon}^{N_{0}}(T)-\eta_{\varepsilon}^{N_{0}}(T-h)\right) \\
& =\int_{0}^{T} \int_{\Omega_{\eta_{\varepsilon}}(t)} \mathbf{f} \cdot \boldsymbol{\phi}_{\varepsilon}+\int_{0}^{T} \int_{\omega} g\left(\eta_{\varepsilon}^{N_{0}}-\left(\eta_{\varepsilon}^{N_{0}}\right)^{-}\right) .
\end{aligned}
$$

The two first terms can be written:

$$
\begin{aligned}
& \left.\left.-\int_{0}^{T} \int_{\Omega_{\eta_{\varepsilon}}(t)} \mathbf{u}_{\varepsilon} \cdot\left(\left(\overline{\mathbf{v}_{\varepsilon}^{\lambda}}\right)_{\sigma}-\left(\overline{\mathbf{v}_{\varepsilon}^{\lambda}}\right)_{\sigma}^{-}\right)-\int_{0}^{T} \int_{\Omega_{\eta_{\varepsilon}}(t)} \mathbf{u}_{\varepsilon} \cdot\left(\overline{\left(\overline{\mathcal{R}_{\alpha}\left(\partial_{t} \eta_{\varepsilon}^{N_{0}}\right.}\right.}\right)-\overline{\mathcal{R}_{\alpha}\left(\partial_{t} \eta_{\varepsilon}^{N_{0}}\right.}\right)^{-}\right) \\
= & -\int_{0}^{T} \int_{\Omega_{\eta_{\varepsilon}}(t)} \mathbf{u}_{\varepsilon} \cdot\left(\overline{\mathbf{u}}_{\varepsilon}-\overline{\mathbf{u}}_{\varepsilon}^{-}\right)-\int_{0}^{T} \int_{\Omega_{\eta_{\varepsilon}}(t)} \mathbf{u}_{\varepsilon} \cdot\left(\overline{\mathcal{R}_{\alpha}\left(\partial_{t} \eta_{\varepsilon}^{h f, N_{0}}\right)}-\overline{\mathcal{R}_{\alpha}\left(\partial_{t} \eta_{\varepsilon}^{h f, N_{0}}\right)}\right) \\
- & \int_{0}^{T} \int_{\Omega_{\eta_{\varepsilon}}(t)} \mathbf{u}_{\varepsilon} \cdot\left(\left(\left(\overline{\mathbf{v}_{\varepsilon}^{\lambda}}\right)_{\sigma}-\overline{\mathbf{v}_{\varepsilon}^{\lambda}}\right)-\left(\left(\overline{\mathbf{v}_{\varepsilon}^{\lambda}}\right)_{\sigma}^{-}-\left(\overline{\mathbf{v}_{\varepsilon}^{\lambda}}\right)^{-}\right)\right)-\int_{0}^{T} \int_{\Omega_{\eta_{\varepsilon}}(t)} \mathbf{u}_{\varepsilon} \cdot\left(\left(\left(\overline{\mathbf{v}_{\varepsilon}^{\lambda}}\right)-\overline{\mathbf{v}}_{\varepsilon}\right)-\left(\left(\overline{\mathbf{v}_{\varepsilon}^{\lambda}}\right)^{-}-\left(\overline{\mathbf{v}}_{\varepsilon}\right)^{-}\right)\right) .
\end{aligned}
$$

$$
\begin{aligned}
& \text { We set } I_{1}=\int_{0}^{T} \int_{\Omega_{\eta_{\varepsilon}}(t)} \mathbf{u}_{\varepsilon} \cdot\left(\overline{\mathbf{u}}_{\varepsilon}-\overline{\mathbf{u}}_{\varepsilon}^{-}\right) . \\
& \begin{aligned}
I_{1} & =-\frac{1}{2} \int_{0}^{T} \int_{\Omega_{\eta_{\varepsilon}}(t)}\left|\mathbf{u}_{\varepsilon}\right|^{2}+\frac{1}{2} \int_{0}^{T} \int_{\Omega_{\eta_{\varepsilon}}(t)}\left|\overline{\mathbf{u}}_{\varepsilon}^{-}\right|^{2}-\frac{1}{2} \int_{0}^{T} \int_{\Omega_{\eta_{\varepsilon}}(t)}\left|\overline{\mathbf{u}}_{\varepsilon}-\overline{\mathbf{u}}_{\varepsilon}^{-}\right|^{2} \\
& =-\frac{1}{2} \int_{0}^{T} \int_{\Omega_{\eta_{\varepsilon}}(t)}\left|\mathbf{u}_{\varepsilon}\right|^{2}+\frac{1}{2} \int_{0}^{T-h} \int_{\Omega_{\eta_{\varepsilon}}(t+h)}\left|\overline{\mathbf{u}}_{\varepsilon}\right|^{2}-\frac{1}{2} \int_{0}^{T} \int_{\Omega_{\eta_{\varepsilon}}(t)}\left|\overline{\mathbf{u}}_{\varepsilon}-\overline{\mathbf{u}}_{\varepsilon}^{-}\right|^{2} \\
& =\frac{1}{2} \int_{0}^{T-h} \int_{\mathcal{C}_{M}}\left(\rho_{\varepsilon}^{+}-\rho_{\varepsilon}\right)\left|\overline{\mathbf{u}}_{\varepsilon}\right|^{2}-\frac{1}{2} \int_{T-h}^{T} \int_{\Omega_{\eta_{\varepsilon}}(t)}\left|\mathbf{u}_{\varepsilon}\right|^{2}-\frac{1}{2} \int_{0}^{T} \int_{\Omega_{\eta_{\varepsilon}}(t)}\left|\overline{\mathbf{u}}_{\varepsilon}-\overline{\mathbf{u}}_{\varepsilon}^{-}\right|^{2} .
\end{aligned}
\end{aligned}
$$

The same argument we use to prove (32) leads to

$$
\int_{0}^{T} \int_{\mathcal{C}_{M}}\left|\rho_{\varepsilon}^{+}-\rho_{\varepsilon}\right|\left|\overline{\mathbf{u}}_{\varepsilon}\right|^{2} \leq C h^{\frac{1}{3}}
$$


This yields

$$
I_{1} \leq C h^{\frac{1}{3}}-\frac{1}{2} \int_{0}^{T} \int_{\Omega_{\eta_{\varepsilon}}(t)}\left|\overline{\mathbf{u}}_{\varepsilon}-\overline{\mathbf{u}}_{\varepsilon}^{-}\right|^{2} .
$$

For the second term of (37) $\left.\int_{0}^{T} \int_{\Omega_{\eta_{\varepsilon}}(t)} \mathbf{u}_{\varepsilon} \cdot \overline{\left(\overline{\mathcal{R}_{\alpha}\left(\partial_{t} \eta_{\varepsilon}^{h f, N_{0}}\right)}\right.}-\overline{\mathcal{R}_{\alpha}\left(\partial_{t} \eta_{\varepsilon}^{h f, N_{0}}\right)}\right)$ we have, taking into account the energy estimate (9),

$$
\begin{aligned}
& \mid \int_{0}^{T} \int_{\Omega_{\eta_{\varepsilon}}(t)} \mathbf{u}_{\varepsilon} \cdot\left(\overline{\mathcal{R}_{\alpha}\left(\partial_{t} \eta_{\varepsilon}^{h f, N_{0}}\right)}-\overline{\mathcal{R}_{\alpha}\left(\partial_{t} \eta_{\varepsilon}^{h f, N_{0}}\right)}-1\right. \\
& \leq C\left\|\mathbf{u}_{\varepsilon}\right\|_{L^{2}\left(0, T ; L^{2}\left(\Omega_{\eta_{\varepsilon}}(t)\right)\right)}\left\|\overline{\mathcal{R}_{\alpha}\left(\partial_{t} \eta_{\varepsilon}^{h f, N_{0}}\right)}\right\|_{L^{2}\left(0, T ; L^{2}\left(\mathcal{C}_{M}\right)\right)} \\
& \quad \leq C\left(\left\|\mathcal{R}_{\alpha}\left(\partial_{t} \eta_{\varepsilon}^{h f, N_{0}}\right)\right\|_{L^{2}\left(0, T ; L^{2}\left(\mathcal{C}_{\alpha}\right)\right)}+\left\|\partial_{t} \eta_{\varepsilon}^{h f, N_{0}}\right\|_{L^{2}\left(0, T ; L^{2}(\omega)\right)}\right) .
\end{aligned}
$$

Thanks to the properties satisfied by $\mathcal{R}_{\alpha}$ and in particular (17) for $s=0$ we have

$$
\left\|\mathcal{R}_{\alpha}\left(\partial_{t} \eta_{\varepsilon}^{h f, N_{0}}\right)\right\|_{L^{2}\left(0, T ; L^{2}\left(\mathcal{C}_{\alpha}\right)\right)} \leq C\left\|\partial_{t} \eta_{\varepsilon}^{h f, N_{0}}\right\|_{L^{2}\left(0, T ; L^{2}(\omega)\right)},
$$

thus we obtain

$$
\mid \int_{0}^{T} \int_{\Omega_{\eta_{\varepsilon}}(t)} \mathbf{u}_{\varepsilon} \cdot \overline{\left(\mathcal{R}_{\alpha}\left(\partial_{t} \eta_{\varepsilon}^{h f, N_{0}}\right)\right.}-\overline{\mathcal{R}_{\alpha}\left(\partial_{t} \eta_{\varepsilon}^{h f, N_{0}}\right)}-1 \leq C\left\|\partial_{t} \eta_{\varepsilon}^{h f, N_{0}}\right\|_{L^{2}\left(0, T ; L^{2}(\omega)\right)} .
$$

The definition of $\partial_{t} \eta_{\varepsilon}^{h f, N_{0}}$ and the fact that $\partial_{t} \eta_{\varepsilon}$ is bounded in $L^{2}\left(0, T ; H^{s}(\omega)\right), s<$ $1 / 2$ independently of $\varepsilon$, imply, remembering (34), that

$\left.\mid \int_{0}^{T} \int_{\Omega_{\eta_{\varepsilon}}(t)} \mathbf{u}_{\varepsilon} \cdot \overline{\left(\mathcal{R}_{\alpha}\left(\partial_{t} \eta_{\varepsilon}^{h f, N_{0}}\right)\right.}-\overline{\mathcal{R}_{\alpha}\left(\partial_{t} \eta_{\varepsilon}^{h f, N_{0}}\right)}-\right) \mid \leq C\left\|\partial_{t} \eta_{\varepsilon}^{h f, N_{0}}\right\|_{L^{2}\left(0, T ; L^{2}(\omega)\right)} \leq C \lambda_{N_{0}}^{-\frac{s}{2}}$.

We have also for the third term of (37):

$$
\begin{gathered}
\mid \int_{0}^{T} \int_{\Omega_{\eta_{\varepsilon}}(t)} \mathbf{u}_{\varepsilon} \cdot\left(\left(\left(\overline{\mathbf{v}_{\varepsilon}^{\lambda}}\right)_{\sigma}-\overline{\mathbf{v}_{\varepsilon}^{\lambda}}\right)-\left(\left(\overline{\mathbf{v}_{\varepsilon}^{\lambda}}\right)_{\sigma}^{-}-\left(\overline{\mathbf{v}_{\varepsilon}^{\lambda}}\right)^{-}\right) \mid\right. \\
\left.\leq 2\left\|\mathbf{u}_{\varepsilon}\right\|_{L^{2}\left(0, T ; L^{2}\left(\Omega_{\eta_{\varepsilon}}(t)\right)\right)} \|\left(\overline{\mathbf{v}_{\varepsilon}^{\lambda}}\right)_{\sigma}-\overline{\mathbf{v}_{\varepsilon}^{\lambda}}\right) \|_{L^{2}\left(0, T ; L^{2}\left(\Omega_{\eta_{\varepsilon}}(t)\right)\right)} \\
\leq(\sigma-1)\left\|\overline{\mathbf{v}_{\varepsilon}^{\lambda}}\right\|_{L^{2}\left(0, T ; H^{1}\left(\Omega_{\eta_{\varepsilon}}(t)\right)\right)} \\
\leq C_{\lambda}(\sigma-1) \leq C_{\lambda} h^{\mu}, \quad 0<\mu<\frac{1}{2}
\end{gathered}
$$

Finally, du to the propreties of the space regularization $\mathbf{v} \mapsto \mathbf{v}^{\lambda}$ the last term of the right hand side of (37) goes to zero, uniformly in $\varepsilon$, as $\lambda$ goes to zero. That writes: $\forall \beta>0$ there exists $\lambda_{0}>0$ such that $\forall \lambda<\lambda_{0}$

$$
\left|\int_{0}^{T} \int_{\Omega_{\eta_{\varepsilon}}(t)} \mathbf{u}_{\varepsilon} \cdot\left(\left(\left(\overline{\mathbf{v}_{\varepsilon}^{\lambda}}\right)-\overline{\mathbf{v}}_{\varepsilon}\right)-\left(\left(\overline{\mathbf{v}_{\varepsilon}^{\lambda}}\right)^{-}-\left(\overline{\mathbf{v}}_{\varepsilon}\right)^{-}\right)\right)\right| \leq C \beta, \quad \forall \varepsilon .
$$


Now we take care of the convective term

$$
I_{2}=\int_{0}^{T} \int_{\Omega_{\eta_{\varepsilon}}(t)}\left(\mathbf{u}_{\varepsilon} \cdot \nabla\right) \mathbf{u}_{\varepsilon} \cdot\left(\int_{t-h}^{t} \boldsymbol{\psi}_{\varepsilon}\right),
$$

with $\boldsymbol{\psi}_{\varepsilon}=\left(\overline{\mathbf{v}_{\varepsilon}^{\lambda}}\right)_{\sigma}+\overline{\mathcal{R}_{\alpha}\left(\partial_{t} \eta_{\varepsilon}^{N_{0}}\right)}$. We have

$$
\begin{aligned}
\left|I_{2}\right| & \leq \frac{1}{2} \int_{0}^{T}\left\|\mathbf{u}_{\varepsilon}\right\|_{L^{4}\left(\Omega_{\eta_{\varepsilon}}(t)\right)}\left\|\nabla \mathbf{u}_{\varepsilon}\right\|_{L^{2}\left(\Omega_{\eta_{\varepsilon}}(t)\right)} \int_{t-h}^{t}\left\|\boldsymbol{\psi}_{\varepsilon}\right\|_{L^{4}\left(\Omega_{\eta_{\varepsilon}}(t)\right)} \\
& \leq \frac{\sqrt{h}}{2} \int_{0}^{T}\left\|\mathbf{u}_{\varepsilon}\right\|_{L^{4}\left(\Omega_{\eta_{\varepsilon}}(t)\right)}\left\|\nabla \mathbf{u}_{\varepsilon}\right\|_{L^{2}\left(\Omega_{\eta_{\varepsilon}}(t)\right)}\left(\int_{t-h}^{t}\left\|\boldsymbol{\psi}_{\varepsilon}\right\|_{L^{4}\left(\Omega_{\eta_{\varepsilon}}(t)\right)}^{2}\right)^{\frac{1}{2}} \\
& \leq C_{N_{0}, \lambda} \sqrt{h} .
\end{aligned}
$$

The next term to consider is $I_{3}=\nu \int_{0}^{T} \int_{\Omega_{\eta_{\varepsilon}}(t)} \nabla \mathbf{u}_{\varepsilon}: \nabla\left(\int_{t-h}^{t} \boldsymbol{\psi}_{\varepsilon}\right)$.

$$
\begin{aligned}
\left|I_{3}\right| & \leq \nu \int_{0}^{T}\left\|\nabla \mathbf{u}_{\varepsilon}\right\|_{L^{2}\left(\Omega_{\eta_{\varepsilon}}(t)\right)} \int_{t-h}^{t}\left\|\nabla \boldsymbol{\psi}_{\varepsilon}\right\|_{L^{2}\left(\Omega_{\eta_{\varepsilon}}(t)\right)} \\
& \leq \nu \sqrt{h} \int_{0}^{T}\left\|\nabla \mathbf{u}_{\varepsilon}\right\|_{L^{2}\left(\Omega_{\eta_{\varepsilon}}(t)\right)}\left(\int_{t-h}^{t}\left\|\nabla \boldsymbol{\psi}_{\varepsilon}\right\|_{L^{2}\left(\Omega_{\eta_{\varepsilon}}(t)\right)}^{2}\right)^{\frac{1}{2}} \\
& \leq C_{N_{0}, \lambda} \sqrt{h} .
\end{aligned}
$$

The term $I_{4}=\int_{\Omega_{\eta_{\varepsilon}}(T)} \mathbf{u}_{\varepsilon}(T) \cdot\left(\int_{T-h}^{T} \boldsymbol{\psi}_{\varepsilon}\right)$ can be estimated as follows:

$$
\begin{aligned}
\left|I_{4}\right| & \leq\left\|\mathbf{u}_{\varepsilon}(T)\right\|_{L^{2}\left(\Omega_{\eta_{\varepsilon}}(T)\right)} \int_{T-h}^{T}\left\|\boldsymbol{\psi}_{\varepsilon}\right\|_{L^{2}\left(\Omega_{\eta_{\varepsilon}}(T)\right)} \\
& \leq \sqrt{h}\left\|\mathbf{u}_{\varepsilon}(T)\right\|_{L^{2}\left(\Omega_{\eta_{\varepsilon}}(T)\right)}\left(\int_{T-h}^{T}\left\|\boldsymbol{\psi}_{\varepsilon}\right\|_{L^{2}\left(\Omega_{\eta_{\varepsilon}}(T)\right)}^{2}\right)^{\frac{1}{2}} \\
& \leq C \sqrt{h}
\end{aligned}
$$

We set $I_{5}=-\int_{0}^{T} \int_{\omega} \partial_{t} \eta_{\varepsilon} \partial_{t}\left(\eta_{\varepsilon}^{N_{0}}-\left(\eta_{\varepsilon}^{N_{0}}\right)^{-}\right)$.

We have thanks to the definition of $\eta_{\varepsilon}^{N_{0}}$

$$
\begin{aligned}
I_{5} & =-\frac{1}{2} \int_{0}^{T} \int_{\omega}\left(\partial_{t} \eta_{\varepsilon}^{N_{0}}\right)^{2}+\frac{1}{2} \int_{0}^{T} \int_{\omega}\left(\left(\partial_{t} \eta_{\varepsilon}^{N_{0}}\right)^{-}\right)^{2}-\frac{1}{2} \int_{0}^{T} \int_{\omega}\left(\partial_{t} \eta_{\varepsilon}^{N_{0}}-\left(\partial_{t} \eta_{\varepsilon}^{N_{0}}\right)^{-}\right)^{2} \\
& =-\frac{1}{2} \int_{T_{-} h}^{T} \int_{\omega}\left(\partial_{t} \eta_{\varepsilon}^{N_{0}}\right)^{2}-\frac{1}{2} \int_{0}^{T} \int_{\omega}\left(\partial_{t} \eta_{\varepsilon}^{N_{0}}-\left(\partial_{t} \eta_{\varepsilon}^{N_{0}}\right)^{-}\right)^{2} \\
& \leq-\frac{1}{2} \int_{0}^{T} \int_{\omega}\left(\partial_{t} \eta_{\varepsilon}^{N_{0}}-\left(\partial_{t} \eta_{\varepsilon}^{N_{0}}\right)^{-}\right)^{2}
\end{aligned}
$$

For the next term we have

$$
\begin{aligned}
\left|I_{6}\right| & =\left|\frac{1}{2} \int_{0}^{T} \int_{\omega}\left(\partial_{t} \eta_{\varepsilon}^{N_{0}}\right)^{2}\left(\eta_{\varepsilon}^{N_{0}}-\left(\eta_{\varepsilon}^{N_{0}}\right)^{-}\right)\right| \\
& \leq \frac{1}{2} \int_{0}^{T}\left\|\partial_{t} \eta_{\varepsilon}^{N_{0}}\right\|_{L^{3}(\omega)}^{2}\left\|\eta_{\varepsilon}^{N_{0}}-\left(\eta_{\varepsilon}^{N_{0}}\right)^{-}\right\|_{L^{3}(\omega)}
\end{aligned}
$$


but, taking into account the continuous imbedding of $H^{s}(\omega), 0 \leq s<1 / 2$ in $L^{3}(\omega)$, and the fact that $\partial_{t} \eta_{\varepsilon}^{N_{0}}$ is bounded uniformly in $\varepsilon$ in $L^{2}\left(0, T, H^{s}(\omega)\right), \forall 0 \leq$ $s<1 / 2$

$$
\left\|\eta_{\varepsilon}^{N_{0}}-\left(\eta_{\varepsilon}^{N_{0}}\right)^{-}\right\|_{L^{3}(\omega)} \leq \int_{t-h}^{t}\left\|\partial_{t} \eta_{\varepsilon}^{N_{0}}\right\|_{L^{3}(\omega)} \leq C \sqrt{h} .
$$

Consequently

$$
\left|I_{6}\right| \leq C \sqrt{h}
$$

The next term to consider is $I_{7}=\int_{0}^{T} \int_{\omega} \Delta \eta_{\varepsilon}^{N_{0}} \Delta\left(\eta_{\varepsilon}^{N_{0}}-\left(\eta_{\varepsilon}^{N_{0}}\right)^{-}\right)$. It can be estimated as follows

$$
\begin{aligned}
\left|I_{7}\right| & \leq \int_{0}^{T}\left\|\Delta \eta_{\varepsilon}^{N_{0}}\right\|_{L^{2}(\omega)} \int_{t-h}^{t}\left\|\Delta \partial_{t} \eta_{\varepsilon}^{N_{0}}\right\|_{L^{2}(\omega)} \\
& \leq \sqrt{h} \int_{0}^{T}\left\|\Delta \eta_{\varepsilon}^{N_{0}}\right\|_{L^{2}(\omega)}\left(\int_{t-h}^{t}\left\|\Delta \partial_{t} \eta_{\varepsilon}^{N_{0}}\right\|_{L^{2}(\omega)}^{2}\right)^{\frac{1}{2}} \\
& \leq C_{N_{0}} \sqrt{h} .
\end{aligned}
$$

The additional viscous term gives for $\varepsilon \leq 1$ :

$$
\begin{aligned}
\left|I_{8}\right| & =\varepsilon\left|\int_{0}^{T} \int_{\omega} \Delta \partial_{t} \eta_{\varepsilon}^{N_{0}} \Delta\left(\eta_{\varepsilon}^{N_{0}}-\left(\eta_{\varepsilon}^{N_{0}}\right)^{-}\right)\right| \\
& \leq \int_{0}^{T}\left\|\Delta \partial_{t} \eta_{\varepsilon}^{N_{0}}\right\|_{L^{2}(\omega)} \int_{t-h}^{t}\left\|\Delta \partial_{t} \eta_{\varepsilon}^{N_{0}}\right\|_{L^{2}(\omega)} \\
& \leq \sqrt{h} \int_{0}^{T}\left\|\Delta \partial_{t} \eta_{\varepsilon}^{N_{0}}\right\|_{L^{2}(\omega)}\left(\int_{t-h}^{t}\left\|\Delta \partial_{t} \eta_{\varepsilon}^{N_{0}}\right\|_{L^{2}(\omega)}^{2}\right)^{\frac{1}{2}} \\
& \leq C_{N_{0}} \sqrt{h} .
\end{aligned}
$$

We set $I_{9}=\int_{\omega} \partial_{t} \eta_{\varepsilon}^{N_{0}}(T)\left(\eta_{\varepsilon}(T)-\eta_{\varepsilon}^{N_{0}}(T-h)\right)$.

$$
\begin{aligned}
\left|I_{9}\right| & =\left\|\partial_{t} \eta_{\varepsilon}(T)\right\|_{L^{2}(\omega)} \int_{T-h}^{T}\left\|\partial_{t} \eta_{\varepsilon}^{N_{0}}\right\|_{L^{2}(\omega)} \\
& \leq C h .
\end{aligned}
$$

Next $I_{10}=\int_{0}^{T} \int_{\Omega_{\eta_{\varepsilon}}(t)} \mathbf{f} \cdot\left(\int_{t-h}^{t} \boldsymbol{\psi}_{\varepsilon}\right)$ and $I_{11}=\int_{0}^{T} \int_{\omega} g\left(\eta_{\varepsilon}^{N_{0}}-\left(\eta_{\varepsilon}^{N_{0}}\right)^{-}\right)$can be estimated respectively by $C \sqrt{h}$ and $C h$.

These last two estimates and estimates (38)-(50) yield, for all $\beta>0$, for $\lambda$ small enough

$\int_{0}^{T} \int_{\Omega_{\eta_{\varepsilon}}(t)}\left|\mathbf{u}_{\varepsilon}-\overline{\mathbf{u}}_{\varepsilon}^{-}\right|^{2}+\int_{0}^{T} \int_{\omega}\left(\partial_{t} \eta_{\varepsilon}^{N_{0}}-\left(\partial_{t} \eta_{\varepsilon}^{N_{0}}\right)^{-}\right)^{2} \leq C_{N_{0}, \lambda} h^{\frac{1}{3}}+C \lambda_{N_{0}}^{-\frac{s}{2}}+C \beta, \forall \varepsilon<\varepsilon_{0}$, with $s<\frac{1}{2}$. Moreover,

$$
\int_{0}^{T} \int_{\omega}\left(\partial_{t} \eta_{\varepsilon}^{h f, N_{0}}-\left(\partial_{t} \eta_{\varepsilon}^{h f, N_{0}}\right)^{-}\right)^{2} \leq C \lambda_{N_{0}}^{-\frac{s}{2}} .
$$


Then for all $\beta>0$, if $\lambda$ is chosen small enough we have

$\int_{0}^{T} \int_{\Omega_{\eta_{\varepsilon}}(t)}\left|\mathbf{u}_{\varepsilon}-\overline{\mathbf{u}}_{\varepsilon}^{-}\right|^{2}+\int_{0}^{T} \int_{\omega}\left(\partial_{t} \eta_{\varepsilon}-\left(\partial_{t} \eta_{\varepsilon}\right)^{-}\right)^{2} \leq C_{N_{0}, \lambda} h^{\frac{1}{3}}+C \lambda_{N_{0}}^{-\frac{s}{2}}+C \beta, \quad \forall \varepsilon<\varepsilon_{0}$.

Thus, choosing $N_{0}$ large enough and $\lambda$ small enough, we obtain that $\exists h_{0}>0$ such that $\forall h \leq h_{0}$

$$
\int_{0}^{T} \int_{\Omega_{\eta_{\varepsilon}}(t)}\left|\mathbf{u}_{\varepsilon}-\overline{\mathbf{u}}_{\varepsilon}^{-}\right|^{2}+\int_{0}^{T} \int_{\omega}\left(\partial_{t} \eta_{\varepsilon}-\left(\partial_{t} \eta_{\varepsilon}\right)^{-}\right)^{2} \leq C \beta, \quad \forall \varepsilon<\varepsilon_{0} .
$$

This proves Lemma 10.

Thanks to Lemma 9, we obtain that $\partial_{t} \eta_{\varepsilon}$ is compact in $L^{2}\left(0, T ; L^{2}(\omega)\right)$ and that $\overline{\mathbf{u}}_{\varepsilon}$ is compact in $L^{2}\left(0, T ; L^{2}\left(\mathcal{C}_{M}\right)\right)$.

We now want to verify the convergences announced in Proposition 1 and to verify that the equality between the structure velocity and the fluid velocity at the interface holds in the limit.

Let $T>0$ such that $\inf _{\varepsilon} \min _{[0, T] \times \bar{\omega}}\left(1+\eta_{\varepsilon}\right) \geq \alpha>0$. We will denote any subsequence of $\left(\eta_{\varepsilon}, \overline{\mathbf{u}}_{\varepsilon}\right)$ by $\left(\eta_{\varepsilon}, \overline{\mathbf{u}}_{\varepsilon}\right)$. Thanks to the energy estimate and to the compactness properties that have just been derived, we have, denoting by $(\eta, \underline{\mathbf{u}})$ the limit of a subsequence of $\left(\eta_{\varepsilon}, \overline{\mathbf{u}}_{\varepsilon}\right)$, the following convergences as $\varepsilon$ goes to zero:

$$
\begin{aligned}
& \eta_{\varepsilon} \quad \rightarrow \quad \eta \quad \text { uniformly in } \quad C^{0}\left([0, T] ; C^{0}(\bar{\omega})\right), \\
& \eta_{\varepsilon} \quad \rightarrow \quad \eta \quad \text { weakly in } \quad L^{2}\left(0, T ; H_{0}^{2}(\omega)\right) \text {, } \\
& \partial_{t} \eta_{\varepsilon} \rightarrow \partial_{t} \eta \quad \text { strongly in } L^{2}\left(0, T ; L^{2}(\omega)\right) \text {, } \\
& \partial_{t} \eta_{\varepsilon} \rightarrow \partial_{t} \eta \quad \text { weakly in } \quad L^{2}\left(0, T ; W^{1-1 / p, p}(\omega)\right), \forall 1<p<2 \text {, } \\
& \partial_{t} \eta_{\varepsilon} \rightarrow \partial_{t} \eta \quad \text { weakly in } \quad L^{2}\left(0, T ; H^{s}(\omega)\right), \forall 0 \leq s<1 / 2 \text {, } \\
& \overline{\mathbf{u}}_{\varepsilon} \quad \rightarrow \quad \underline{\mathbf{u}} \quad \text { strongly in } \quad L^{2}\left(0, T ; L^{2}\left(\mathcal{C}_{M}\right)\right) \text {, } \\
& \rho_{\varepsilon} \mathbf{u}_{\varepsilon} \rightarrow \rho \underline{\mathbf{u}} \quad \text { strongly in } \quad L^{2}\left(0, T ; L^{2}\left(\mathcal{C}_{M}\right)\right) \text {. }
\end{aligned}
$$

Moreover $\rho_{\varepsilon} \nabla \mathbf{u}_{\varepsilon}$ tends to some $\mathbf{z}$ weakly in $L^{2}\left(0, T ; L^{2}\left(\mathcal{C}_{M}\right)\right)$ as $\varepsilon$ goes to zero. It is easy to verify that, since $\eta_{\varepsilon} \rightarrow \eta$ in $C^{0}([0, T] \times \bar{\omega})$, that $\mathbf{z}=0$ in $\widehat{\mathcal{C}_{M}} \backslash \widehat{\Omega}_{\eta}$ and $\left.\mathbf{z}\right|_{\widehat{\Omega}_{\eta}}=\nabla\left(\left.\underline{\mathbf{u}}\right|_{\widehat{\Omega}_{\eta}}\right)$. Thus

$$
\rho_{\varepsilon} \nabla \mathbf{u}_{\varepsilon} \rightarrow \rho \nabla\left(\left.\underline{\mathbf{u}}\right|_{\widehat{\Omega}_{\eta}}\right) \text { in } L^{2}\left(0, T ; L^{2}\left(\mathcal{C}_{M}\right)\right),
$$

Note also that $\underline{\mathbf{u}}=\left(0,0, \partial_{t} \eta\right)^{T}$ in $\widehat{\mathcal{C}_{M}} \backslash \widehat{\Omega}_{\eta}$, thus $\underline{\mathbf{u}}=\overline{\mathbf{u}}$ by setting $\mathbf{u}=\underline{\mathbf{u}} \widehat{\Omega}_{\eta}$. Next we take care of the equality

$$
\mathbf{u}_{\varepsilon}\left(t, x, y, 1+\eta_{\varepsilon}(t, x, y)\right)=\left(0,0, \partial_{t} \eta_{\varepsilon}(t, x, y)\right)^{T}
$$

on $(0, T) \times \omega$. The right hand side converges to $\left(0,0, \partial_{t} \eta\right)^{T}$ strongly in $L^{2}\left(0, T ; L^{2}(\omega)\right)$. The left hand side is the trace of the function $\mathbf{w}_{\varepsilon}(t, x, y, z)=\mathbf{u}_{\varepsilon}(t, x, y, z(1+$ $\left.\eta_{\varepsilon}(t, x, y)\right)$ on $z=1$ and $\mathbf{w}_{\varepsilon}$ converges strongly in $L^{2}\left(0, T ; L^{2}\left(\mathcal{C}_{1}\right)\right)$ and weakly 
in $L^{2}\left(0, T ; W^{1, p}\left(\mathcal{C}_{1}\right)\right), \forall 1<p<2$ to $\mathbf{u}(t, x,, y, z(1+\eta(t, x, y))$. Hence by the continuity of the trace mapping on $z=1$, we have, for a. e. $t$,

$$
\mathbf{u}(t, x, y, 1+\eta(t, x, y))=\left(0,0, \partial_{t} \eta(t, x, y)\right)^{T} \text { on } \omega .
$$

This ends the proof of Proposition 1.

\section{Passage to the limit - Proof of Theorem 2}

Next we pass to the limit in the weak formulation:

$$
\begin{gathered}
\int_{\Omega_{\eta_{\varepsilon}}(t)} \mathbf{u}_{\varepsilon}(t) \cdot \boldsymbol{\phi}_{\varepsilon}(t)-\int_{0}^{t} \int_{\Omega_{\eta_{\varepsilon}}(s)} \mathbf{u}_{\varepsilon} \cdot \partial_{t} \boldsymbol{\phi}_{\varepsilon}+\nu \int_{0}^{t} \int_{\Omega_{\eta_{\varepsilon}}(s)} \nabla \mathbf{u}_{\varepsilon}: \nabla \boldsymbol{\phi}_{\varepsilon} \\
+\int_{0}^{t} \int_{\Omega_{\eta_{\varepsilon}}(s)}\left(\mathbf{u}_{\varepsilon} \cdot \nabla\right) \mathbf{u}_{\varepsilon} \cdot \boldsymbol{\phi}_{\varepsilon}-\int_{0}^{t} \int_{\omega}\left(\partial_{t} \eta_{\varepsilon}\right)^{2} b+\int_{\omega} \partial_{t} \eta_{\varepsilon}(t) b(t) \\
-\int_{0}^{t} \int_{\omega} \partial_{t} \eta_{\varepsilon} \partial_{t} b+\varepsilon \int_{0}^{t} \int_{\omega} \Delta \partial_{t} \eta_{\varepsilon} \Delta b+\int_{0}^{t} \int_{\omega} \Delta \eta_{\varepsilon} \Delta b \\
=\int_{0}^{t} \int_{\Omega_{\eta_{\varepsilon}}(t)} \mathbf{f} \cdot \boldsymbol{\phi}_{\varepsilon}+\int_{0}^{t} \int_{\omega} g b+\int_{\Omega(0)} \mathbf{u}_{I} \cdot \boldsymbol{\phi}_{\varepsilon}(0)+\int_{\omega} \dot{\eta}_{I} b(0),
\end{gathered}
$$

for a. e. $t$ and for all $\left(\phi_{\varepsilon}, b\right) \in\left(V_{\eta_{\varepsilon}} \cap H^{1}\left(\widehat{\Omega}_{\eta_{\varepsilon}}\right)\right) \times\left(L^{2}\left(0, T ; H_{0}^{2}(\omega)\right) \times H^{1}\left(0, T ; L^{2}(\omega)\right)\right)$ such that $\phi_{\varepsilon}\left(t, x, y, 1+\eta_{\varepsilon}(t, x, y)\right)=(0,0, b(t, x, y))^{T},(t, x, y) \in[0, T] \times \omega$.

The fluid test functions should depend on $\varepsilon$. However, it is sufficient to consider a dense family of test functions and it can be chosen independent of $\varepsilon$ and admissible for any $\varepsilon$ small enough.

First we consider test functions of the form $\left(\phi^{0}, 0\right)$, such that $\phi^{0}$ belongs to $\mathcal{D}\left(\cup_{t \in[0, T]}\{t\} \times \Omega_{\eta}(t)\right)$ and $\operatorname{div} \phi^{0}=0$. These test functions satisfy the property that $\phi^{0}(t, \cdot) \in \mathcal{D}\left(\Omega_{\eta}(t)\right), \forall t$. For $\varepsilon$ small enough, since $\eta_{\varepsilon}$ converges uniformly to $\eta, \phi^{0} \in \mathcal{D}\left(\cup_{t \in[0, T]}\{t\} \times \Omega_{\eta_{\varepsilon}}(t)\right)$.

The second pair of test functions we consider is $\left(\phi^{1}, b\right)$ where $b$ belongs to $L^{2}\left(0, T ; H_{0}^{2}(\omega)\right) \cap H^{1}\left(0, T ; L^{2}(\omega)\right)$ with $\int_{\omega} b=0$ and for a. e. $t$

$$
\phi^{1}=\mid \begin{aligned}
& (0,0, b)^{T} \text { in } \widehat{\mathcal{C}_{M}} \backslash \widehat{C}_{\alpha} \\
& \mathcal{R}\left(0,0, \frac{z}{\alpha} b\right)^{T} \text { in } \widehat{C}_{\alpha},
\end{aligned}
$$

where $\widehat{C}_{\alpha}=(0, T) \times \mathcal{C}_{\alpha}$ and $\mathcal{R}$ is a linear lifting operator from $\left\{\mathbf{w} \in H^{\frac{1}{2}}\left(\partial C_{\alpha}\right) ; \int_{\partial C_{\alpha}} \mathbf{w} \cdot \mathbf{n}=0\right\}$ onto $\left\{\mathbf{v} \in H^{1}\left(C_{\alpha}\right) ; \operatorname{div}(\mathbf{v})=0\right\}$. We have easily that $\phi^{1}$ belongs to $L^{2}\left(0, T ; H^{1}\left(\mathcal{C}_{M}\right)\right)$ and $\operatorname{div}\left(\phi^{1}\right)=0$. Moreover since $\min _{[0, T] \times \bar{\omega}}\left(1+\eta_{\varepsilon}\right) \geq \alpha, \phi^{1}(t, x, y, 1+$ $\left.\eta_{\varepsilon}(t, x, y)\right)=(0,0, b(t, x, y))^{T}$ on $(0, T) \times(\omega), \forall \varepsilon$. Furthermore we can choose the linear operator $\mathcal{R}$ such that

$$
\left\|\mathcal{R}\left(0,0, \frac{z b}{\alpha}\right)^{T}\right\|_{L^{2}(\omega \times(0, \alpha))} \leq C\|b\|_{L^{2}(\omega)} .
$$


It can be done by solving a Stokes problem in $\omega \times(0, \alpha)$. Indeed this type of inequality can be obtained thanks to a transposition argument and relies on a $H^{2} \times H^{1}$ regularity result for the Stokes problem which is true here since $\omega \times(0, \alpha)$ is a convex set (see [9] for the regularity result for the Stokes problem). Thus if $\mathcal{R}$ is chosen such that (52) holds, we deduce that, for a. e. $t \in I \subset \subset$ $(0, T)$, and for $h$ small enough

$$
\left\|\mathcal{R}\left(0,0, \frac{z b}{\alpha}\right)^{T}(t)-\mathcal{R}\left(0,0, \frac{z b}{\alpha}\right)^{T}(t+h)\right\|_{L^{2}(\omega \times(0, \alpha))} \leq C\|b(t)-b(t+h)\|_{L^{2}(\omega)} .
$$

Since $\partial_{t} b \in L^{2}\left(0, T ; L^{2}(\omega)\right)$ this implies that $\partial_{t} \mathcal{R}\left(0,0, \frac{z b}{\alpha}\right)^{T} \in L^{2}\left(0, T ; L^{2}\left(\mathcal{C}_{\alpha}\right)\right)$ and that $\partial_{t} \phi^{1} \in L^{2}\left(0, T ; L^{2}\left(\mathcal{C}_{M}\right)\right)$. Consequently $\left(\phi^{1}, b\right)$ is a pair of admissible test functions for all $\varepsilon$.

With both type of test functions, it is easy to pass to the limit in the weak formulation as $\varepsilon$ goes to zero. Since the considered family of test functions is dense in the set of functions $(\phi, b) \in\left(V_{\eta} \cap H^{1}\left(\widehat{\Omega}_{\eta}\right)\right) \times\left(L^{2}\left(0, T ; H_{0}^{2}(\omega)\right) \times\right.$ $\left.H^{1}\left(0, T ; L^{2}(\omega)\right)\right)$ such that $\phi(t, x, y, 1+\eta(t, x, y))=(0,0, b(t, x, y))^{T},(t, x, y) \in$ $[0, T] \times \omega$, we obtain the existence of one weak solution on $(0, T)$ of $(22)$ that moreover satisfies the energy estimate (27) by passing to the limit as $\varepsilon$ tends to zero in (9).

Eventually, we show that we can extend the solution, as long as we have $\min _{[0, T] \times \bar{\omega}}(1+\eta)>0$. We do exactly as in [4], but for the sake of completness we reproduce the proof here. We build an increasing sequence of times $\left(T_{k}\right)_{k \geq 1}$ as follows. First we choose a time $T_{1}>0$ such that there exists a weak solution up to $T_{1}$, with $m_{1}=\min _{\left[0, T_{1}\right] \times \bar{\omega}}(1+\eta)>0$. Possibly changing slightly $T_{1}$, we may moreover assume that $\eta\left(T_{1}\right) \in H_{0}^{2}(\omega), \partial_{t} \eta\left(T_{1}\right) \in L^{2}(\omega)$ and $\mathbf{u}\left(T_{1}\right) \in L^{2}\left(\Omega_{\eta}\left(T_{1}\right)\right)$ (since this is true for almost each time).

Now, let $k \geq 1$ and assume that we have built a solution up to some time $T_{k}$, with $m_{k}=\min _{\left[0, T_{k}\right] \times \bar{\omega}}(1+\eta)>0$. Our construction allows us to build an extension of our solution, on some time interval starting from $T_{k}$. Thanks to the energy estimate (27) (see also (9)), we have for $s \geq T_{k}$ for any $0<\lambda<\frac{3}{4}$

$$
1+\eta(s) \geq 1+\eta\left(T_{k}\right)-\left(s-T_{k}\right)^{\lambda} C\left(T_{k}, s\right) \geq m_{k}-\left(s-T_{k}\right)^{\lambda} C\left(T_{k}, s\right),
$$

with

$$
\begin{aligned}
C\left(T_{k}, s\right)=\tilde{C}\left(\left\|\mathbf{u}\left(T_{k}\right)\right\|_{L^{2}\left(\Omega_{\eta_{I}}\right)},\left\|\eta\left(T_{k}\right)\right\|_{H_{0}^{2}(\omega)},\left\|\partial_{t} \eta\left(T_{k}\right)\right\|_{L_{0}^{2}(\omega)},\right. \\
\left.\int_{T_{k}}^{s} \exp (s-u)\left(\|\mathbf{f}\|_{L^{2}\left(\Omega_{\eta}(u)\right)}(u)+\|g\|_{L^{2}(\omega)}(u)\right) d u\right),
\end{aligned}
$$

where $\tilde{C}$ is positive and nondecreasing with respect to its arguments, and $C\left(T_{k}, s\right) \leq C(0, s)$. This a priori estimate shows that if we let

$$
\tau_{k}=\min \left\{1,\left(m_{k} / 2 C\left(T_{k}, T_{k}+1\right)\right)^{\frac{1}{\lambda}}\right\},
$$


we can build a solution starting from $\mathbf{u}\left(T_{k}\right)$ and $\eta\left(T_{k}\right), \partial_{t} \eta\left(T_{k}\right)$ up to the time $T_{k}+\tau_{k}$ (this corresponds to choosing $\alpha=m_{k} / 2$ in the construction of the solution). The time $T_{k+1}$ is choosen close to $T_{k}+\tau_{k}$ (in $\left[T_{k}+\tau_{k} / 2, T_{k}+\tau_{k}\right]$ ), in order to have also $\eta\left(T_{k}\right) \in H_{0}^{2}(\omega), \partial_{t} \eta\left(T_{k+1}\right) \in L^{2}(\omega)$ and $\mathbf{u}\left(T_{k+1}\right) \in L^{2}\left(\Omega_{\eta}\left(T_{k+1}\right)\right)$.

If the sequence $\left(T_{k}\right)_{k \geq 1}$ is infinite we let $T^{*}=\sup _{k} T_{k}$. If $T<+\infty$, it must be that $m=\min _{\left[0, T^{*}\right] \times \bar{\omega}}(1+\eta)=0$. Otherwise, we have $m_{k} \geq m$ for all $k$, hence $\tau_{k} \geq \min \left\{1,\left(m / 2 C\left(0, T^{*}\right)\right)^{\frac{1}{\lambda}}\right\}>0$. But $T_{k+1}-T_{k} \geq \tau_{k} / 2$ and goes to zero, a contradiction. This achieves the proof of the theorem.

\section{Conclusion}

We have proved the existence of at least one weak solution for a three-dimensional fluid-plate interaction problem without any (artificial) viscosity of the structure.

\section{References}

[1] R. A. Adams. Sobolev spaces. Academic Press [A subsidiary of Harcourt Brace Jovanovich, Publishers], New York-London, 1975. Pure and Applied Mathematics, Vol. 65.

[2] M. Boulakia. Existence of weak solutions for the motion of an elastic structure in an incompressible viscous fluid. C. R. Math. Acad. Sci. Paris, 336(12):985-990, 2003.

[3] H. Brezis. Analyse fonctionnelle. Masson, Paris, 1983. Théorie et applications. [Theory and applications].

[4] Antonin Chambolle, Benoît Desjardins, Maria J. Esteban, and Céline Grandmont. Existence of weak solutions for the unsteady interaction of a viscous fluid with an elastic plate. J. Math. Fluid Mech., 7(3):368-404, 2005 .

[5] C. Conca, J. San Martín H., and M. Tucsnak. Existence of solutions for the equations modelling the motion of a rigid body in a viscous fluid. Comm. Partial Differential Equations, 25(5-6):1019-1042, 2000.

[6] Daniel Coutand and Steve Shkoller. Motion of an elastic solid inside an incompressible viscous fluid. Arch. Ration. Mech. Anal., 176(1):25-102, 2005.

[7] Daniel Coutand and Steve Shkoller. The interaction between quasilinear elastodynamics and the Navier-Stokes equations. Arch. Ration. Mech. Anal., 179(3):303-352, 2006. 
[8] H. B. da Veiga. On the existence of strong solutions to a coupled fluidstructure evolution problem. J. Math. Fluid Mech., 6(1):21-52, 2004.

[9] M. Dauge. Stationary Stokes and Navier-Stokes systems on two- or threedimensional domains with corners. I. Linearized equations. SIAM J. Math. Anal., 20(1):74-97, 1989.

[10] B. Desjardins and M. J. Esteban. Existence of weak solutions for the motion of rigid bodies in a viscous fluid. Arch. Ration. Mech. Anal., 146(1):59-71, 1999.

[11] B. Desjardins and M. J. Esteban. On weak solutions for fluid-rigid structure interaction: compressible and incompressible models. Comm. Partial Differential Equations, 25(7-8):1399-1413, 2000.

[12] B. Desjardins, M. J. Esteban, C. Grandmont, and P. Le Tallec. Weak solutions for a fluid-structure interaction model. Rev. Mat. Complut., 14(2):523-538, 2001.

[13] E. Feireisl. On the motion of rigid bodies in a viscous compressible fluid. Arch. Rational Mech. Anal., 167(4):281-308, 2003.

[14] Giovanni P. Galdi. On the motion of a rigid body in a viscous liquid: a mathematical analysis with applications. In Handbook of mathematical fluid dynamics, Vol. I, pages 653-791. North-Holland, Amsterdam, 2002.

[15] V. Girault and P-A. Raviart. Finite element methods for Navier-Stokes equations. Springer-Verlag, Berlin, 1986. Theory and algorithms.

[16] C. Grandmont and Y. Maday. Existence for an unsteady fluid-structure interaction problem. M2AN Math. Model. Numer. Anal., 34(3):609-636, 2000 .

[17] M. D. Gunzburger, H-C. Lee, and G. A. Seregin. Global existence of weak solutions for viscous incompressible flows around a moving rigid body in three dimensions. J. Math. Fluid Mech., 2(3):219-266, 2000.

[18] K.-H. Hoffmann and V. N. Starovoitov. On a motion of a solid body in a viscous fluid. Two-dimensional case. Adv. Math. Sci. Appl., 9(2):633-648, 1999.

[19] J.-L. Lions and E. Magenes. Non-homogeneous boundary value problems and applications. Vol. I, Vol. I. Springer-Verlag, New York, 1972. Translated from the French by P. Kenneth, Die Grundlehren der mathematischen Wissenschaften, Band 181. 
[20] J.-L. Lions and E. Magenes. Non-homogeneous boundary value problems and applications. Vol. I, Vol. II. Springer-Verlag, New York, 1972. Translated from the French by P. Kenneth, Die Grundlehren der mathematischen Wissenschaften, Band 181.

[21] J. A. San Martín, V. Starovoitov, and M. Tucsnak. Global weak solutions for the two-dimensional motion of several rigid bodies in an incompressible viscous fluid. Arch. Ration. Mech. Anal., 161(2):113-147, 2002.

[22] D. Serre. Chute libre d'un solide dans un fluide visqueux incompressible. Existence. Japan J. Appl. Math., 4(1):99-110, 1987.

[23] J. Simon. Compact sets in the space $L^{p}(0, T ; B)$. Ann. Mat. Pura Appl. (4), 146:65-96, 1987.

[24] T. Takahashi and M. Tucsnak. Global strong solutions for the two dimensional motion of a rigid body in a viscous fluid. J. Math. Fluid Mech., $6(1): 53-77,2004$

[25] Takéo Takahashi. Analysis of strong solutions for the equations modeling the motion of a rigid-fluid system in a bounded domain. Adv. Differential Equations, 8(12):1499-1532, 2003.

[26] R. Temam. Navier-Stokes equations. Theory and numerical analysis. North-Holland Publishing Co., Amsterdam, 1977. Studies in Mathematics and its Applications, Vol. 2. 